Portland State University

PDXScholar

Spring 6-13-2018

\title{
Development of a Direct Aqueous Synthetic Route for the Production of Elemental Bismuth Nanoparticles
}

Colin Jon Hiatt

Portland State University

Follow this and additional works at: https://pdxscholar.library.pdx.edu/open_access_etds

Part of the Chemistry Commons

Let us know how access to this document benefits you.

Recommended Citation

Hiatt, Colin Jon, "Development of a Direct Aqueous Synthetic Route for the Production of Elemental Bismuth Nanoparticles" (2018). Dissertations and Theses. Paper 4446.

https://doi.org/10.15760/etd.6330

This Thesis is brought to you for free and open access. It has been accepted for inclusion in Dissertations and Theses by an authorized administrator of PDXScholar. Please contact us if we can make this document more accessible: pdxscholar@pdx.edu. 
Development of a Direct Aqueous Synthetic Route for the Production of Elemental Bismuth Nanoparticles

by

Colin Jon Hiatt

A thesis submitted in partial fulfillment of the requirements for the degree of

Master of Science

in Chemistry

Thesis Committee: Andrea Goforth, Chair David Stuart Theresa McCormick Shankar Rananavare

Portland State University 2018 


\section{Abstract}

Bismuth nanoparticles (Bi NPs) for use as an X-ray contrast material have gained significant traction in recent years due to the high atomic number and generally accepted biological tolerance of bismuth. However, to be considered a viable candidate for use in this application, water solubility is a necessity, which poses a challenge, since bismuth tends to readily oxidize. For this reason, research into the direct aqueous synthesis of Bi NPs is still in its infancy and can be very difficult, as described in Chapter 1 . The remaining chapters of the thesis describe the direct aqueous synthesis of Bi NPs starting from a water-soluble bismuth tartrate $\left(\mathrm{Bi}_{\mathrm{x}}(\mathrm{D}-\mathrm{TA})_{\mathrm{y}}\right)$ precursor. Syntheses were carried out in an aerobic environment using a variety of $\mathrm{pH}$ and temperature conditions, from biologically compatible and inexpensive chemical reagents.

Chapter 2 describes initial studies that sought to use glucose as the reducing agent and its oxidation product, gluconic acid, as the surface-stabilizing species. These studies showed glucose to be ineffective as a reducing agent for bismuth, as reactions progressed slowly and resulting particles lacked size and shape uniformity. The addition of a co-reductant, borane morpholine, was observed to result in an increased reaction rate, which yielded particles that exhibited improved size and shape uniformity. However, due to lack of surface stabilization, resulting particles were often observed to undergo oxidative dissolution upon quenching of the reaction. To better stabilize particle surfaces, glucose was replaced with 300 MW poly(ethylene glycol) (PEG 300). This change resulted in an overall decrease in 
the rate of reaction. The majority of syntheses using PEG as a surfactant resulted in Bi NPs that were equally unstable, as particle colloids were often observed to dissolve when quenching the reaction. For samples in which oxidative dissolution did not occur, the resulting Bi NPs were observed to be crystalline, aggregated nanostructures lacking any size or shape uniformity. For samples that underwent immediate oxidative dissolution and were afterwards left in a closed container absent of light for $\sim 96$ hours at room temperature, regrowth of Bi NPs was observed, where particle growth was thought to occur through a seed-mediated pathway. Through this seed-mediated growth method, resulting Bi NPs were observed to have significantly improved size and shape uniformity, as well as aqueous colloidal stability.

Chapter 3 describes additional syntheses that yielded highly stable and relatively size uniform aqueous Bi NPs, which were prepared by chemical reduction of the same $\mathrm{Bi}_{\mathrm{x}}(\mathrm{D}-\mathrm{TA})_{\mathrm{y}}$ precursor. In these studies, hexamethylenediamine (HMD) was used as the surface-stabilizing agent in place of PEG 300. Analysis via FTIR revealed the presence of the ligand tartrate, which facilitated a simple acid/base titration method of particle isolation. With the addition of $\mathrm{HCl}$, particle colloids would flocculate, allowing for ease of separation from the reaction medium by centrifugation. The Bi NPs could then be re-dispersed in aqueous solution with the addition of $\mathrm{NaOH}$. 


\section{Dedication}

For my mama and papa. You're unyielding belief in me keeps me going. 


\section{Acknowledgements}

First and foremost, I would like to thank my research advisor, Dr. Andrea Goforth. She has been instrumental in my development as a scientist, teaching me to keep my research more focused and less exploratory, amongst other things. This has been a long row to hoe and without your support and guidance, I'm sure this would have been an even more difficult process.

Secondly, I'd like to thank Dr. David Tyler at the University of Oregon for giving me a chance and taking me on as an intern at the beginning of my scientific career, and for an additional two summers as a paid research assistant, as well. You gave me much needed exposure to chemical research that informed my decision to pursue graduate school. Additionally, I'd like to give a shout out to Dr. Alexander J. Kendall, who was my mentor during my time as an undergraduate researcher at the $\mathrm{U}$ of $\mathrm{O}$. Many weekends and late nights in the lab, fueled by coffee and followed by beer and D\&D made that experience even more memorable.

I would also like to thank my committee members, Dr. Theresa McCormick, Dr. David Stuart, and Dr. Shankar Rananavare. You all have been guiding me since I started my tenure at PSU and have been an inspiring force to my success in this program. Whether it was talking research or personal matters, you all have always kept an open door and an open ear.

Additionally, I would like to thank my lab mates, past and present. You all are amazing people and exceptional chemists. Extra special thanks go out to Dr. ShengKuei Chiu and Hayden Winter for TEM advice and support. This leads me to thank those responsible for maintaining the facilities that allow me to blast samples with 
electrons to get beautiful images of my particles. Thank you Dr. Tony Chen, Greg Baty, and the rest of the support staff at the Center for Electron Microscopy and Nanofabrication (CEMN).

Of course I should take this opportunity to thank my family, especially my niece and nephew who think I'm the coolest and smartest person on the planet. Bless their hearts and sweet ignorance.

Finally, to the dopest kween I've had the pleasure to know. Your unwavering drive and confidence in pursuit of your own goals inspires me daily to turn my limitations into opportunity and to strive to be better each day. While it may not seem like it at times, your encouragement toward me keeps me pressing ever forward. I could not envision a better partner and can't wait to see what we achieve next. 


\section{Table of Contents}

ABSTRACT

DEDICATION

ACKNOWLEDGEMENTS iv

LIST OF FIGURES viii

LIST OF SCHEMES ix

CHAPTER 1: THE CHEMISTRY OF BISMUTH NANOMATERIALS 1

1.1 MOTIVATION AND BACKGROUND 1

1.1.1 Bismuth Nanomaterial Applications 1

1.1.2 Bi NPs as an X-Ray Contrast Agent as the Main Motivation 4

1.2 NANOPARTICLE SYNTHESIS IN GENERAL 8

1.2.1 Nucleation and Growth Kinetics 8

$\begin{array}{lr}\text { 1.2.2 Preventing Aggregation } & 10\end{array}$

1.2.3 Thermodynamic Synthetic Considerations 13

1.3 SYNTHESIS OF NANOCRYSTALLINE BISMUTH PARTICLES 16

1.3.1 Organic Synthetic Strategies 16

1.3.2 Aqueous Synthetic Strategies $\quad 19$

1.4 CONCLUSIONS 23

CHAPTER 2: EMPLOYING REDOX ACTIVE SURFACE STABILIZING AGENTS IN THE DIRECT SYNTHESIS OF COLLOIDALLY STABLE AQUEOUS BISMUTH NANOPARTICLES $\quad 25$

2.1 ABSTRACT 25

2.2 INTRODUCTION 26

2.3 EXPERIMENTAL 31

2.3.1 Materials 31

$\begin{array}{ll}\text { 2.3.2 Synthesis } & 31\end{array}$

2.3.3 Bismuth Nanoparticle Characterization $\quad 34$

2.4 RESULTS AND DISCUSSION 36

2.4.1 pH-Dependent Synthesis of Bismuth Nanoparticles Using Glucose as a Reductant $\quad 36$

2.4.2 Borane Morpholine-Assisted Synthesis of Bismuth Nanoparticles $\quad 43$

2.4.3 Synthesis of Bismuth Nanoparticles Using PEG 300 as a Surface-

Stabilizing Agent $\quad 46$

2.5 CONCLUSIONS 53

CHAPTER 3: ONE-STEP SYNTHESIS OF TARTRATE-STABILIZED AQUEOUS COLLOIDAL BISMUTH NANOPARTICLES 55

3.1 ABSTRACT $\quad 55$

3.2 INTRODUCTION 55 
3.3 EXPERIMENTAL $\quad 57$

3.3.1 Materials

3.3.2 Synthesis $\quad 57$

3.3.3 Bismuth Nanoparticle Characterization $\quad 58$

3.4 RESULTS AND DISCUSSION $\quad 60$

3.5 CONCLUSIONS 65

TERMINAL REFERENCES

$\begin{array}{ll}\text { APPENDIX } & 77\end{array}$ 


\section{List of Figures}

Figures:

Page

Figure 1.1 Evaluation of X-ray attenuation of Bi NPs.

Figure 1.2 LaMer model describing the formation of monodispersed 9

particles in three distinct stages: I, prenucleation; II, nucleation; and III, particle growth.

Figure 1.3 Illustrations depicting several common ways to stabilize particle surfaces to protect the particles from aggregation.

Figure 1.4 Uniform Bi NPs generated by different organic and highenergy synthetic protocols.

Figure 1.5 Bi NPs generated from aqueous protocols yielding 20 hydrophobic particles with random morphologies and large size distributions.

Figure 1.6 Bi NPs synthesized via an aqueous pH-dependent route. 22

Figure 1.7 Bi NPs synthesized in PPD with the surface stabilizer and 24 co-reductant $\alpha$-D-glucose.

Figure 2.1 Characterization of Bi NPs synthesized using glucose as $\quad 41$ the sole reductant.

Figure 2.2 Characterization of $\mathrm{Bi}_{2} \mathrm{O}_{3}$ NPs precipitated from reaction 43 before addition of glucose.

Figure 2.3 Characterization of Bi NPs synthesized using borane 45 morpholine as a co-reductant.

Figure 2.4 Characterization of Bi NPs synthesized using borane 48 morpholine as the sole reductant and PEG 300 as the surface-stabilizing agent.

Figure 2.5 Characterization of Bi NPs synthesized through an incubation route, where particle growth occurs via a seedmediated mechanism.

Figure 2.6 Characterization of product obtained in incubation control reactions.

Figure 3.1 Characterization of Bi NPs synthesized using borane 61 morpholine as the sole reductant in the presence of HMD.

Figure 3.2 Surface characterization of Bi NPs synthesized in the 63 presence of HMD.

$\begin{array}{lll}\text { Figure S1 Representative FTIR spectrum of Bi NPs synthesized using } & 77\end{array}$ glucose as the sole reducing agent.

Figure S2 FTIR spectrum of Bi NPs synthesized in the presence of PEG 300 using borane morpholine as the reducing agent.

$\begin{array}{lll}\text { Figure S3 FTIR spectrum of particle colloid that underwent oxidative } \quad 79 & 79\end{array}$ dissolution and regrew through incubation of the dissolved colloid. 


\section{List of Schemes}

Schemes:

Page

Scheme 1.1 Reaction scheme for the reductive coupling of nitroarenes to azo compounds using Bi NPs as catalysts.

Scheme 1.2 Reaction scheme of the reduction of 4-nitrophenol to 4aminophenol with $\mathrm{NaBH}_{4}$, where Bi NPs catalyze the reaction.

Scheme 2.1 Isomeric aqueous equilibrium of glucose and redox halfreaction of D-glucose.

Scheme 2.2 Scheme depicting the reaction of D-(-)-tartaric acid with bismuth (III).

Scheme 3.1 Scheme depicting the method with which Bi NPs synthesized in the presence of HMD were isolated, using a combination chemical/physical approach. 


\section{Chapter 1 - The Chemistry of Bismuth Nanomaterials}

\subsection{Motivation and Background}

The chemistry of bismuth nanomaterials is a relatively new and burgeoning area of study, where nanoparticles comprised of crystalline bismuth cores are of particular interest. This is due to their incredibly diverse range of potential applications, such as in heterogeneous and homogeneous catalysis, ${ }^{1-3}$ as lubricating oil additives, ${ }^{4}$ as thermoelectric materials, ${ }^{5-7}$ as well as for biomedical X-ray imaging agents. ${ }^{8-15}$ Particularly for use in biomedical X-ray imaging, synthesized particles must be water soluble and biocompatible. While there do exist direct aqueous synthetic preparations for bismuth nanoparticles (Bi NPs), as discussed in Section 1.3.2, the majority of synthetic Bi NP literature focuses on organic, air-free, and high-energy techniques, as these methods tend to generate highly uniform and sizetunable NPs, as discussed in Section 1.3.1. The following is a discussion on the current state of Bi NP chemistry and NP chemistry in general, that will demonstrate the progress that has been made, while highlighting the challenges still yet to be met.

\subsubsection{Bismuth Nanomaterial Applications}

In recent years, Bi NPs have been shown to be an effective photocatalyst, catalyzing the oxidation and removal of NO gas. ${ }^{1} \mathrm{NO}$ removal is shown to be at $9.6 \%$ with respect to the initial NO concentration, using $280 \mathrm{~nm}$ light irradiation, without the use of Bi NPs, but increases significantly to $46.0 \%$ when Bi NPs are introduced to the system. This is thought to be due to the formation of superoxide $\left({ }^{\cdot} \mathrm{O}_{2}^{-}\right)$and a hydroxyl radical $(\cdot \mathrm{OH})$ upon photoirradiation of the Bi NPs in the presence of 
adsorbed $\mathrm{O}_{2}$ and water. These reactive oxygen species are thought to go on to react with $\mathrm{NO}$, resulting in the formation of $\mathrm{NO}_{3}$. Additionally, the Bi NPs maintained stable photocatalytic activity over five consecutive cycles, and showed high durability in photocatalytic activity for more than five continuous hours of irradiation, demonstrating the robustness of the particles under catalytic conditions.

Bi NPs have additionally been shown to be efficient catalysts in organic synthesis. For the reductive coupling of nitroarenes to form azo-compounds, Bi NPs have shown great utility as a catalyst. ${ }^{2}$ The catalytic cycle goes through a variety of intermediary steps, where the Bi NP catalysts are oxidized, then regenerated through reduction with sodium borohydride $\left(\mathrm{NaBH}_{4}\right)$, forming nitroso and azoxyarene intermediates, and ending in the resulting azo compound (Scheme 1.1).

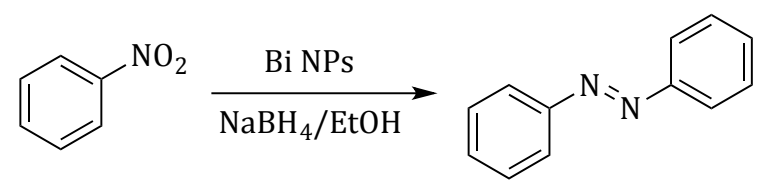

Scheme 1.1: Reaction scheme for the reductive coupling of nitroarenes to azo compounds using Bi NPs as catalysts. Adapted from [2].

With substituted arenes, azo compound yields ranged between 62-96\%, depending on the functional group, showing slowly diminishing yields with each catalytic cycle. Sterically bulky groups and strongly electron-donating groups produced the lowest yields, while small electron-withdrawing groups or weakly electron-donating groups gave the best yields.

Bi NPs have also demonstrated efficiency in catalyzing the chemical reduction of 4-nitrophenol (4-NP) using $\mathrm{NaBH}_{4}$ (Scheme 1.2). ${ }^{3}$ This particular 
reaction is often used to evaluate the catalytic performance of metal nanoparticles. While the reaction of 4-NP with $\mathrm{NaBH}_{4}$ is a thermodynamically favored one, the kinetic barrier associated with the energy of activation makes the reaction difficult to carry out. To that end, the nanoparticles act as a "shuttle" to relay electrons between $\mathrm{BH}_{4}^{-}$and 4-NP.

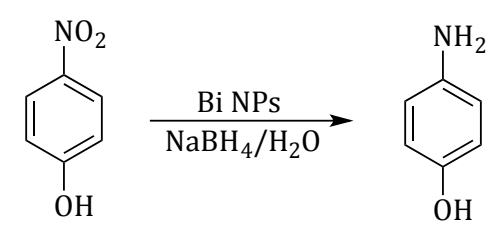

Scheme 1.2: Reaction scheme of the reduction of 4-nitrophenol to 4-aminophenol with $\mathrm{NaBH}_{4}$, where Bi NPs catalyze the reaction. Adapted from [3].

In addition to being an effective catalyst material, elemental bismuth has also been shown to be effective as an additive in lubricating oils. ${ }^{4}$ Bismuth as a lubricant additive has been shown to decrease friction coefficients, in general, by six-times the original friction coefficient when kept below $200^{\circ} \mathrm{C}$. However, when the lubricated surfaces were elevated above the melting temperature of the bismuth powder $\left(271^{\circ} \mathrm{C}\right)$, a layer of oxide developed, which resulted in an increase of the friction coefficient.

Bismuth nanomaterials have also been shown to be an effective thermoelectric material. Thermoelectric materials generate electricity in the presence of a temperature differential on either side of the material. Conversely, they can generate a temperature differential with an applied current. The efficiency of this conversion is typically evaluated through the following expression:

$$
Z T=\frac{S^{2} \sigma}{\kappa} T
$$


where $S$ represents thermoelectric power, which is the thermoelectric voltage $\left(V_{p}\right)$ generated per degree temperature difference, $\sigma$ is electrical conductivity, $\kappa$ is the thermal conductivity, and $T$ is temperature. ${ }^{6}$ In order to achieve high efficiency in a thermoelectric material, $Z T$ (the thermoelectric figure of merit) must be maximized. To that end, bismuth presents several distinct advantages. Bulk bismuth has a large thermoelectric power $\left(S=50\right.$ to $100 \mu \mathrm{VK}^{-1}$ ) and a small thermal conductivity ( $\kappa=8$ $\left.\mathrm{W} \mathrm{m}^{-1} \mathrm{~K}^{-1}\right)$. However, it is theorized that $S$ can be enhanced significantly when bismuth crystallite domains are reduced to the nanoscale, due to a semimetal-tosemiconductor transition that is brought on by the effects of quantum-confinement. ${ }^{7}$

\subsubsection{Bi NPs as an X-ray Contrast Agent as the Main Motivation}

While bismuth nanomaterials have been shown to be quite versatile, demonstrating utility in a broad scope of applications, none have been more investigated than the use of Bi NPs as X-ray contrast agents (XCAs). Nanoparticles as XCAs are of particular interest given that they are comprised of a dense network of atoms and have diameters up to $100 \mathrm{~nm}$, which contributes to their overall ability to attenuate X-ray radiation. Attenuation by a material decreases the intensity of incident radiation due to absorption and/or scattering of photons. This property is largely governed by the X-ray attenuation coefficient $(\mu)$ of the material, ${ }^{16}$ which is represented by the following expression:

$$
\mu=\frac{\rho Z^{4}}{A E^{3}}
$$

where $\rho$ is the material density, $Z$ is the atomic number of the X-ray absorbing atoms, $A$ is the atomic mass, and $E$ is the energy of the incident X-ray radiation. 
Given the direct proportionality of material density and atomic number to X-ray absorption, high-density materials that are comprised of high atomic number elements are the most attractive candidates for XCAs.

A variety of elements and compounds have been investigated for use as nanoparticle XCAs. Studies have shown that inorganic nanoparticle XCAs tend to have characteristically longer circulation times and better chemical stabilities as compared to small molecules, characteristics which have enabled studies aimed at site-directed imaging.17,18 PEGylated erbium-doped ytterbium oxide nanoparticles and nanoparticles containing a tantalum oxide core, as well as a variety of other materials have been investigated for such applications. ${ }^{18-20}$ However, the majority of the research concerning nanoparticle XCAs has focused on gold nanoparticles $\mathrm{Au}$ NPs), owing to the highly studied nature of their syntheses and applications. Au NPs are readily synthesized and exhibit many desirable optical and electronic properties, lending to their continued research uses in biomedicine, as well as components for a wide variety of other applications. ${ }^{17,21-25}$ However, gold is expensive and there still remains some risk of toxicity due to the tendency of Au NPs to bioaccumulate. ${ }^{17}$

Bismuth is an attractive alternative to gold, as bismuth has a greater atomic number than gold ( $\mathrm{Bi}, Z=83 ; \mathrm{Au}, Z=79$ ), while gold has a density that is nearly three times that of bismuth. When examining the two parameters that are directly correlated with the effectiveness of an X-ray absorbing material ( $Z^{4}$ and $\rho$ ), gold and bismuth come out within an order of magnitude of each other, making them 
comparable XCA candidates. Unlike Au NPs, however, elemental Bi NPs undergo oxidation and hydrolysis in water, decomposing over time into smaller molecular or ionic bismuth(III) species, which can assist with avoiding bioaccumulation and possibly lead to better systematic clearance.

Many studies have been carried out to determine the efficacy of Bi NPs as XCAs. One such study was carried out in our lab, where the X-ray attenuation of glucose-coated Bi NPs was examined as a function of concentration (Figure 1.1a). ${ }^{8}$ As seen in the plot of attenuation versus effective bismuth concentration, there is a strong linear correlation between bismuth concentration and measured attenuation. Additionally, when measured at varying X-ray tube voltages, there is very little difference in measured attenuation. When the rate of attenuation of Bi NPs was evaluated against that of free bismuth(III) ions and an aqueous iodine standard (Figure 1.1b), both free bismuth(III) ions and Bi NPs outperformed the iodine standard, with statistically insignificant differences measured between the bismuth(III) ions and elemental Bi NPs.

Additional studies were done to ascertain the biological compatibility of the glucose-coated Bi NPs. Cell viability studies were carried out on the J774A.1 murine macrophage and HeLa human cervix adenocarcinoma cell lines. After one hour of incubation, there was no observed decrease in cell viability for either cell line at any of the tested Bi NPs concentrations. After 24 hours of incubation, macrophage cell viability showed a notable decrease, with a measured $\mathrm{LD}_{50}$ of $50 \mu \mathrm{g} \mathrm{mL}^{-1}$, while the HeLa cell line showed no signs of decreased cell viability. While the macrophage cell 
line did show decreased cell viability over a period of 24 hours, these studies do suggest relatively good biological compatibility.

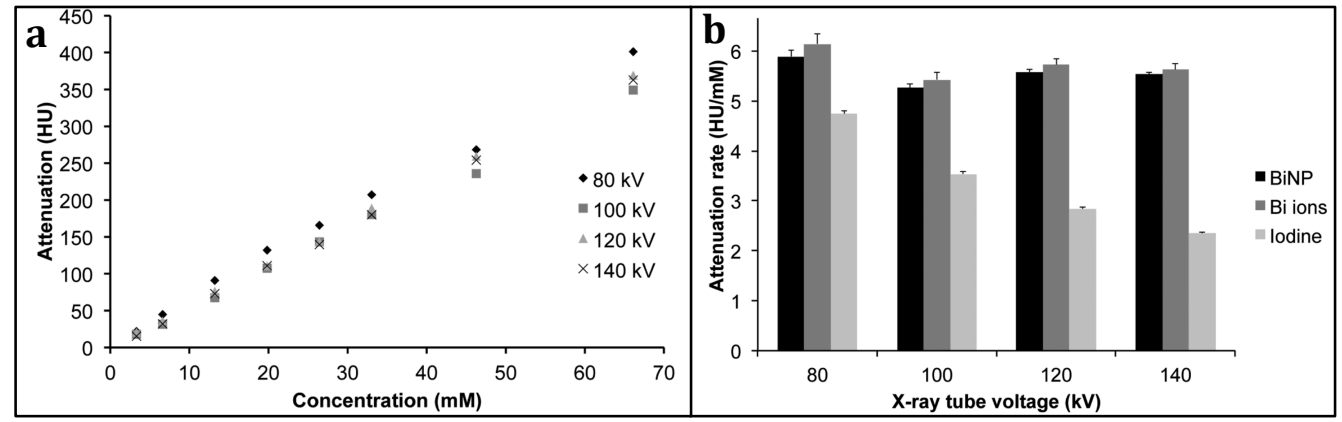

Figure 1.1: Evaluation of X-ray attenuation of Bi NPs. a) X-ray attenuation of Bi NPs measured as a function of concentration, evaluated at four clinically relevant X-ray tube voltages. b) Attenuation rates of Bi NPs, bismuth(III) ions, and an aqueous iodine standard versus X-ray tube voltage. Reproduced with permission from ref. 8, copyright 2014 American Chemical Society.

Z. Li and coworkers were able to further demonstrate the biological compatibility of the glucose-coated Bi NPs. ${ }^{9}$ Nanomaterials that can be utilized for diagnostics, as well as therapeutics, aptly named theranostic agents, are highly desired in the treatment of cancer, since they are an "all-in one" tool for cancer diagnosis and treatment. By functionalizing the glucose-capped Bi NPs with poly(ethylene glycol), the researchers were able to demonstrate their use as an effective XCA, as well as a competent photothermal therapeutic agent. With enhanced biocompatibility and longer circulation times, injected solutions of Bi NPs could reach tumor sites for imaging and photothermal ablation of tumors, effectively killing tumor cells in vivo. 
While there have been great strides made in demonstrating the effectiveness of Bi NPs as biocompatible XCAs, there still remains many challenges. One specific challenge is in the development of a direct aqueous synthesis that generates Bi NPs that are both hydrophilic and uniform in size and shape. These challenges will be addressed in the following sections.

\subsection{Nanoparticle Synthesis in General}

The generation of nanoparticles, specifically those of uniform size, composition, and morphology, is of significant importance, given that many properties that these materials hold are dictated by these parameters. Uniform size, composition, and morphology of a colloidal particle system allows for the assessment of optical, magnetic, electronic, adsorptive, and catalytic properties, to name a few, which can then be related back to particle size and/or shape. Obtaining uniformity in a colloidal particle system, however, is a delicate and challenging feat. Several requirements must be met in order to achieve such uniformity, including: a) separation of nanoparticle nucleation and growth stages, as well as, b) the prevention of aggregation. ${ }^{26}$ There are several other requirements that are of importance; however, these two are pre-requisite concerns to all others and are the focus of this discussion.

\subsubsection{Nucleation and Growth Kinetics}

The first requirement, that separation of nucleation and growth be well resolved, can be understood in terms of the LaMer model (Figure 1.2). ${ }^{26}$ In this model, LaMer describes the overall particle formation process in three distinct stages. In the first stage, pre-nucleation (Stage I), the production of the desired 
precursor species occurs in solution, and the concentration of this precursor increases over time. Stage II begins when the precursor reaches a critical concentration $\left(\mathrm{C}_{\text {crit }}\right)$, or the minimum concentration at which nucleation can occur. During this stage, the rate of precursor formation is overcome by the rate of nanoparticle nuclei formation. If the rate of nucleation is effectively infinite, no further nucleation can occur. This point is marked by a local maximum in precursor concentration, after which time the concentration of precursor is decreased back to $\mathrm{C}_{\text {crit, }}$ marking the end of the nucleation event. Following nucleation is the growth stage (Stage III), a process that is typically diffusion controlled (i.e., limited by diffusion of the monomer to the newly formed nuclei). Particle growth proceeds until the precursor concentration reaches the equilibrium solubility concentration of precursor with respect to the bulk solid $\left(\mathrm{C}_{\text {sol }}\right)$.

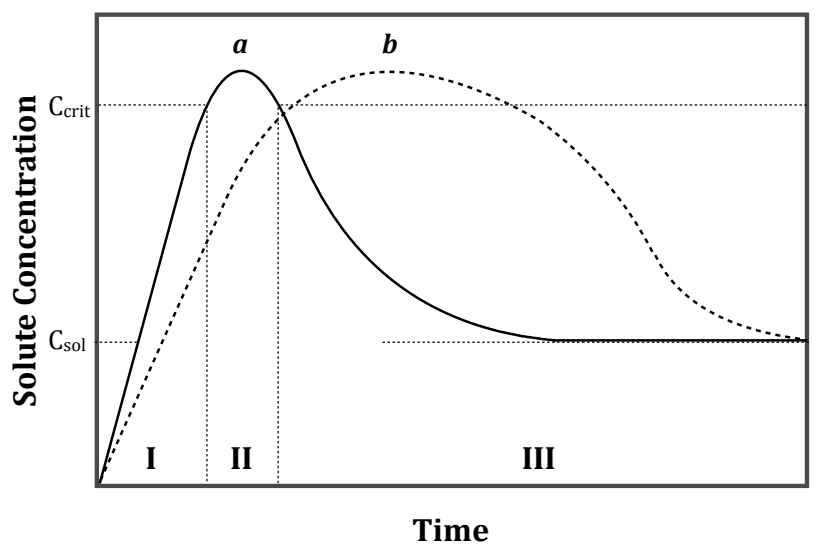

Figure 1.2: LaMer model describing the formation of monodispersed particles in three distinct stages: I, prenucleation; II, nucleation; and III, particle growth. Trace (a) demonstrates an ideal progression through the three stages, while (b) demonstrates a less than ideal progression. Adapted from [26]. 
Achieving a single, rapid nucleation event, followed by a slow period of growth (Figure 1.2a), is of the utmost importance to obtaining colloidal inorganic nanoparticles that are uniform in shape and size. If the nucleation event occurs over an extended period of time (Figure 1.2b), precursor species are unevenly consumed, with nuclei being formed at varying times, ultimately leading to a variety of growth rates on the formed nuclei.

There are a number of techniques by which to separate nucleation from growth. One such technique is to introduce seed crystals into a solution of monomer at a relatively low solute concentration, below $\mathrm{C}_{\text {crit. }}$. With the addition of seed crystals that serve as nucleation sites for further reduction, these seeds can affect directional crystallographic growth, as well as the rate at which crystal growth occurs. In this way, the nucleation and particle growth steps can be effectively isolated as two individual synthetic steps. ${ }^{27}$ Additionally, modification of $\mathrm{pH}$, dilution, addition of a complexing agent, or a quick change in temperature can also affect the separation of nucleation and growth and will be discussed in more detail, as specific reaction parameters are discussed.

\subsubsection{Preventing Aggregation}

The second requirement for obtaining colloidally stable inorganic nanoparticles of uniform size and shape is the prevention of aggregation. Aggregation occurs when two or more particles come in contact with one another, adhering to each other irreversibly. Particles with nanometer-sized domains have elevated surface energies, given their high surface area-to-volume ratio. This tends to lead to either aggregation or dissolution of these particles, the latter in favor of 
the growth of larger, more stable particles, also referred to as Ostwald ripening. The degree to which aggregation or dissolution occur can be minimized in several different ways: by use of an electric double-layer (Figure 1.3a), a solid-state or gellike network (Figure 1.3b), or steric surface-stabilizing agents (Figure 1.3c).

The use of an electric double-layer is an effective method for preventing aggregation, whereby charged particles exert a repulsive force against one another, which is dictated by the zeta potential (the potential difference between the bulk solvent and the stationary layer of solvent molecules attached to the dispersed particle, or the surface of shear ${ }^{28}$ ) and the Debye length (the distance at which an electric field of charge $Q$ is shielded by the ions in a solution ${ }^{29}$ ) of each particle. To impart a particle with an electric double-layer, syntheses are typically carried out in solutions with elevated ionic strength.

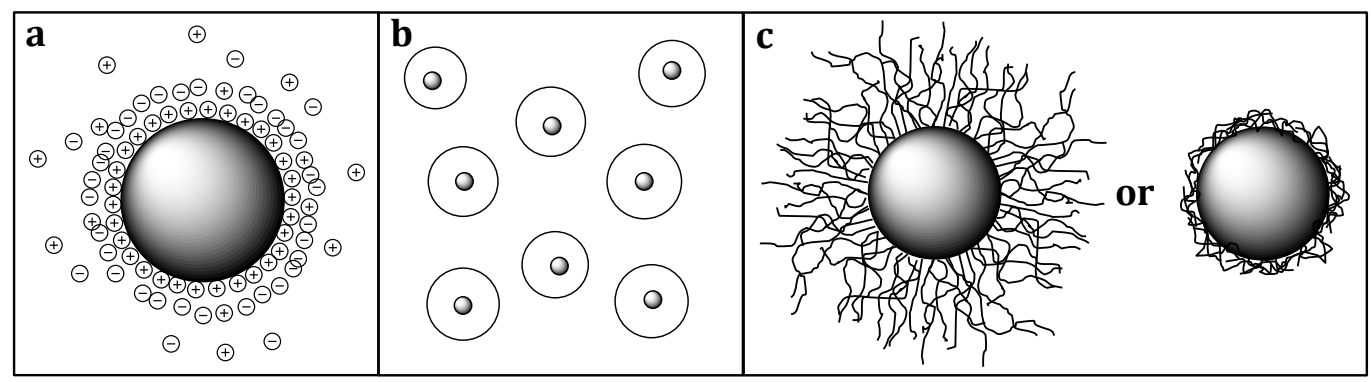

Figure 1.3: Illustrations depicting several common ways to stabilize particle surfaces to protect the particles from aggregation: a) using an electronic double-layer to stabilize particle surfaces; b) nanoparticles that are grown in a solid-state or gel-like network, keeping the particles separated during nucleation and growth stages; and c) nanoparticles stabilized with the use of polymeric or small molecular surface-stabilizing species.

Alternatively, solid-state or gel-like networks formed pre-nucleation can isolate the individual nuclei that form, such that interaction between particles is 
significantly minimized. As an example, our lab developed a synthesis for silicon nanoparticles (Si NPs) that starts with the hydrolysis and polycondensation of trichlorosilane $\left(\mathrm{HSiCl}_{3}\right)$, which forms a hydridosilicate polymer of stoichiometry $\left(\mathrm{HSiO}_{1.5}\right) \mathrm{n} \cdot{ }^{30}$ Annealing of the polymer at $1100^{\circ} \mathrm{C}$ under flowing $\mathrm{N}_{2}$ results in the formation of nanocrystalline Si domains encased in an amorphous silicon dioxide $\left(\mathrm{SiO}_{2}\right)$ matrix, which can subsequently be liberated through an aqueous hydrofluoric acid (HF) etch, leaving hydride-terminated Si NPs.

However, quite possibly the most popular and effective method for preventing aggregation is the use of steric surface-stabilizing agents. These can come in many different forms, from small molecular species to large polymeric species that are adsorbed onto the surface of the particles. Weak bond formation with such species ultimately lowers the total energy of the particle surface.

Apart from being used to stabilize particle surfaces, thus protecting against aggregation, surface-stabilizing agents can also be employed to impart morphological control to growing colloidal particles. In certain cases, some capping agents will preferentially adsorb to specific crystal lattice planes, with a lower extent of binding to other crystallographic planes. Because of this, anisotropic growth can occur due to limiting growth on the crystal plane where binding is the strongest, thereby promoting growth on the crystal planes where binding is weakest.

As an example, it has been demonstrated that alkylphosphonic acid molecules bind to most crystallographic planes in cadmium selenide quantum dots (CdSe QDs) with the exception of the [001] plane. In this way, by altering the 
relative ratios of alkylphosphonic acid to trioctylphosphine oxide surfactants during the period of growth and by slow addition of excess CdSe monomer, CdSe nanorods can be preferentially formed. ${ }^{31,32}$ Similarly, directional growth of the $\mathrm{TiO}_{2}$ anatase crystal structure through surfactant mediation has been demonstrated by the use of a carboxylic acid surfactant that preferentially binds to the higher energy [001] crystal plane. Growth on the [101] crystal plane occurs and allows for the formation of bullet, diamond, rod, and branched structures, depending on the concentration of carboxylic acid..$^{33}$

\subsubsection{Thermodynamic Synthetic Considerations}

To date, a wide variety of methods have been developed for the generation of uniform nanoparticles that reside within two different classifications of approach. Top-down approaches start from bulk material and produce particles through methods such as plasma atomization, aerosol thermolysis, or vapor phase deposition. ${ }^{34-36}$ These are typically physical methods that rely on breaking down the bulk material in order to access particles on the nanoscale and usually require large amounts of energy. Bottom-up techniques, on the other hand, involve the nucleation and isolation of particles from solution using soluble molecular or ionic precursors. In general, these methods are the most versatile, economical, and accessible approaches to generating particles of uniform size and shape. Bottom-up techniques have the ability to generate much more versatile colloids, with regards to their physical characteristics, by modification of synthetic parameters such as the concentration of reactant species, temperature, $\mathrm{pH}$, choice of reducing agent, surface-stabilizing species used, et cetera. While solution synthesis of particles lends 
itself to versatility and ease of preparation, there are a number of factors in reaction conditions that must be considered.

The first, and foremost, condition to consider is the thermodynamic favorability of the reduction of the oxidized metal (or semi-metal) precursor species $\left(\mathrm{M}^{\mathrm{n}+}\right)$. In generating reduced metal species $\left(\mathrm{M}^{0}\right)$ that are the monomers for metal particle formation, the oxidized metallic species undergoes reduction by the following mechanism:

$$
m M^{n+}+n R e d \rightarrow m M^{0}+n \operatorname{Red}^{+}
$$

where Red and $\operatorname{Red}^{+}$are the reduced and oxidized forms of the reducing agent. The magnitude of the difference in reduction potentials in the two half reactions $\left(E_{\text {cell }}\right)$ dictates the extent to which this reaction will occur, as described by the equilibrium constant $(K)$, which is related to the potential difference as follows:

$$
\ln K=\frac{n F E_{c e l l}}{R T}
$$

where $\mathrm{n}$ is the number of electrons transferred in Equation 3, F is the Faraday constant, $\mathrm{R}$ is the universal gas constant, and $\mathrm{T}$ is the absolute temperature. The redox reaction occurs to this extent in order to minimize the free energy $(\Delta G)$, which is related to the potential difference by the following expression:

$$
\Delta G=-n F E_{c e l l}
$$

where a positive $E_{\text {cell }}$ results in a thermodynamically spontaneous reaction, favoring the reduced metal species. With this in mind, the difference in reduction potential between the reducing agent and the metallic species should, ideally, be above the 
range of $0.3-0.4 \mathrm{~V}$ to be of any practical use, as anything lower will result in a reaction that does not proceed, to a large extent, to reduced metal products. ${ }^{37}$

When selecting a thermodynamically appropriate reducing agent for a particular metal species, there are several other parameters to keep in mind. First is the chemical composition of the oxidized metal species, which includes both the metal ions and the ligands bound to the metal ions. Reduction potentials for uncomplexed metal ions versus complexed metal ions can vary significantly. For complexed metal ions, the reduction potential varies as a function of the complex stability, in general, decreasing as complex stability increases. Therefore, complexed metal ions can present thermodynamic limitations in the choice of reducing agents, but they may also provide a kinetic advantage, where strategic manipulations of the metal complex chemistry can aid in tailoring the rate of reactivity of a given synthetic system. In this way, selection of a reducing agent, specific to a particular metal complex, is vital to the success of that reaction.

While modifying a reaction system through metal ion complexation and reducing agent selection is incredibly useful, it is not the only means with which to affect a change in the redox potential of the system. The acidity (or basicity) of a reaction system can also play a major role, given that most aqueous redox reactions involve either $\mathrm{H}^{+}$or ${ }^{-} \mathrm{OH}$ ions. As a result, $E_{\text {cell }}$ manipulations can be carried out by modification of $\mathrm{pH}$ as described by the Nernst equation:

$$
E_{\text {cell }}=E_{\text {cell }}^{o}-\frac{R T}{n F} \ln Q
$$


where $E_{c e l l,}^{o}$ is the standard electrochemical cell potential and $Q$ is the reaction quotient. In aqueous systems, metal ion complexation and $\mathrm{pH}$ modification are almost always used together to control metal oxidation states and precursor composition, which is often described in potential/pH, or Pourbaix, diagrams. By constructing one of these diagrams for a specific metal-reducing agent system, one can predict the thermodynamic conditions appropriate for metal nanoparticle formation and stabilization.

\subsection{Synthesis of Nanocrystalline Bismuth Particles}

In most cases, the synthesis of elemental nanoparticles in aqueous media presents one of the easiest and most viable approaches in nanoparticle synthesis, due to the generally high solubility of the metal salt precursors and common reducing agent species (e.g., sodium borohydride, amine boranes, citrate, etc.) in water. The synthesis of Bi NPs in aqueous media, on the other hand, presents a significant challenge. Bismuth, one of the elements that make up the pnictogen family, has a very strong affinity for chalcogen species such as oxygen and sulfur. Additionally, common bismuth salts are only sparingly soluble in water and require strongly acidic environments for increased aqueous solubility. Furthermore, uncomplexed bismuth(III) has a relatively low reduction potential, $+0.308 \mathrm{~V}$ versus the normal hydrogen electrode (NHE), ${ }^{38}$ making the reduced form susceptible to oxidation in air and water. As such, many synthetic protocols developed for the generation of Bi NPs take place under air-free conditions, typically employing 
deoxygenated organic solvents. ${ }^{39-42}$ Alternately, others utilize a reducing environment generated under high temperature and pressure conditions, such as in solvothermal reduction or in vapor-flow condensation. . $^{43} 49$

\subsubsection{Organic Synthetic Strategies}

As an example, oxidized bismuth (III) thiolate complexes have been used as precursors to Bi NPs. J. S. Son et al. ${ }^{39}$ generated highly monodispersed Bi NPs by the chemical reduction of a highly reactive bismuth dodecanethiolate precursor species in octadecene, using tri- $n$-octyl phosphine (TOP) as the reducing species at $80^{\circ} \mathrm{C}$. By modifying temperature and colloid aging times, they were able to generate Bi NPs of varying sizes, from $6-27 \mathrm{~nm}$, that were highly uniform in size and shape (Figure $1.4 \mathrm{a})$.
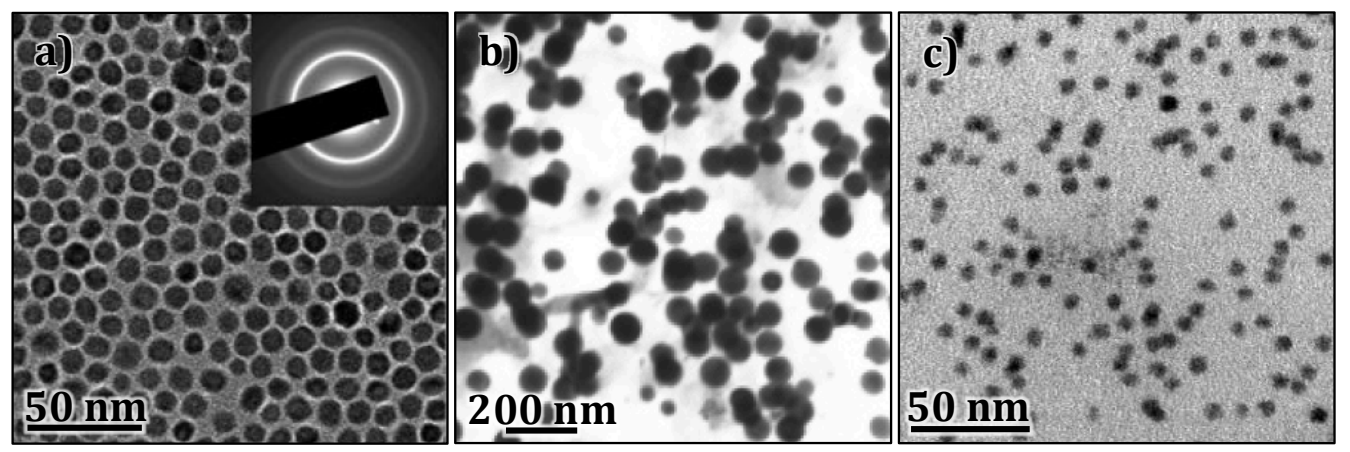

Figure 1.4: Uniform Bi NPs generated by different organic and high-energy synthetic protocols. a) $\mathrm{Bi}$ NPs synthesized through the chemical reduction of bismuth dodecanethiolate with TOP generating monodispersed particles in the range of $6-27 \mathrm{~nm}$ in size, depending on aging temperature and time. Reproduced with permission from ref. 39, copyright 2010 John Wiley and Sons; b) Bi NPs synthesized by the solvothermal reduction of sodium bismuthate in ethylene glycol with varying quantities of acetone to affect size in the range of $10-200 \mathrm{~nm}$. Reproduced with permission from ref. 42, copyright 2004 
American Chemical Society. c) Bi NPs synthesized by thermal decomposition of $\mathrm{Bi}\left[\mathrm{N}\left(\mathrm{SiMe}_{3}\right)_{2}\right]_{3}$ in the presence of $\mathrm{Na}\left[\mathrm{N}\left(\mathrm{SiMe}_{3}\right)_{2}\right]$ generating particles with an average diameter of $7.1 \mathrm{~nm} \pm 0.8 \mathrm{~nm}$. Reproduced with permission from ref. 43, copyright 2008 American Chemical Society.

Alternatively, solvothermal reduction is another common technique for generating monodispersed Bi NPs in an organic solvent. Solvothermal reduction is a method in which metal nanoparticle precursor species are subjected to elevated temperatures and pressures, typically between $100-1000^{\circ} \mathrm{C}$ and $1-10,000$ atm, respectively, enabling precursor interaction and affecting the resulting geometry and size of the target nanomaterial. ${ }^{50}$ Specifically, solvothermal reduction in polyol solvents, such as ethylene or propylene glycol, has a threefold advantage, in that the polyol is the solvent, the reducing agent, and the surface stabilizing species. For example, J. Wang et al. ${ }^{42}$ demonstrated a method for preparing Bi NPs from a sodium bismuthate $\left(\mathrm{NaBiO}_{3}\right)$ precursor in ethylene glycol, that range in size from 10 - $200 \mathrm{~nm}$, depending on the relative ratios of ethylene glycol to acetone co-solvent used (Figure 1.4b). These particles could then later be used as seeds for the preparation of single crystalline nanowires and belts using poly(vinyl pyrolidone) (PVP) to promote and direct growth of the nanowires or belts.

One of the more notable syntheses of Bi NPs comes from F. Wang et al., ${ }^{43}$ which involves the thermal decomposition of the bismuth precursor $\mathrm{Bi}\left[\mathrm{N}\left(\mathrm{SiMe}_{3}\right)_{2}\right]_{3}$ in a mixture of $\mathrm{Na}\left[\mathrm{N}\left(\mathrm{SiMe}_{3}\right)_{2}\right]$, combined with a $25 \mathrm{wt} \%$ polymer solution of poly(1hexadecene $\left.)_{0.67-c o-(1-v i n y l p y r r o l i d i n o n e}\right)_{0.33}$ in 1,3-diisopropylbenzene (polymerDIPB solution) under $\mathrm{O}_{2}$-free $\mathrm{N}_{2}$ (g). The reaction is accomplished in a Schlenk tube 
in a temperature-controlled oil bath at $180^{\circ} \mathrm{C}$ with stirring to generate a deep black colloid after 17 hours. By varying the amount of $\mathrm{Na}\left[\mathrm{N}\left(\mathrm{SiMe}_{3}\right)_{2}\right]$ used in the synthesis, average particle sizes could range from 3.3 to $173.1 \mathrm{~nm}$. However, a typical synthesis generates very uniform spherical particles with an average diameter of $7.1 \pm 0.8 \mathrm{~nm}$ (Figure 1.4c). These particles could then be used as seeds to generate nanorods, nanoribbons, or nanoplates of a single particle width.

While the particles generated in these protocols are size tunable and tend to exhibit high size and shape uniformity, they are typically hydrophobic, such that a ligand exchange would need to be carried out in order to enable water solubility. In the case of polyol syntheses, while these approaches do generate water-soluble nanoparticles, a more direct aqueous-phase approach is desired. A more direct aqueous-phase route would provide greater diversity in choice of surface-stabilizing species, which would then expand the ability to functionalize and tune the particles for biological applications. Such a route would also ideally improve size and morphological homogeneity, which is a general short-coming of polyol reactions. However, given the difficult nature of synthesizing Bi NPs in water, there are only a handful of direct aqueous Bi NP syntheses reported in the literature.

\subsubsection{Aqueous Synthetic Strategies}

Y. Wang et al. ${ }^{51}$ presented an aqueous synthesis of Bi NPs involving chemical reduction of bismuth chloride $\left(\mathrm{BiCl}_{3}\right)$ using hydrazine hydrate $\left(\mathrm{N}_{2} \mathrm{H}_{4} \cdot \mathrm{H}_{2} \mathrm{O}\right)$ as a reducing agent and sodium oleate $(\mathrm{SOA})$ as the surface-stabilizing species, at a 1:20 $\mathrm{BiCl}_{3}: \mathrm{SOA}$ weight ratio. Particles from this synthesis are ultimately hydrophobic, but 
with highly uniform spherical morphology. In a typical synthesis, the resulting particles range in size from $25-40 \mathrm{~nm}$ (Figure 1.5a).

In another aqueous protocol, D. Ma et al. ${ }^{52}$ synthesized Bi NPs from a soluble bismuth tartrate precursor, using sodium hypophosphite $\left(\mathrm{NaH}_{2} \mathrm{PO}_{2}\right)$ as a mild reducing agent in the presence of SOA. The resulting Bi NPs from this protocol are also hydrophobic, but with random morphologies. Furthermore, these particles have a relatively large size distribution, with particle sizes ranging from $10-50 \mathrm{~nm}$ in a single sample (Figure 1.5b).
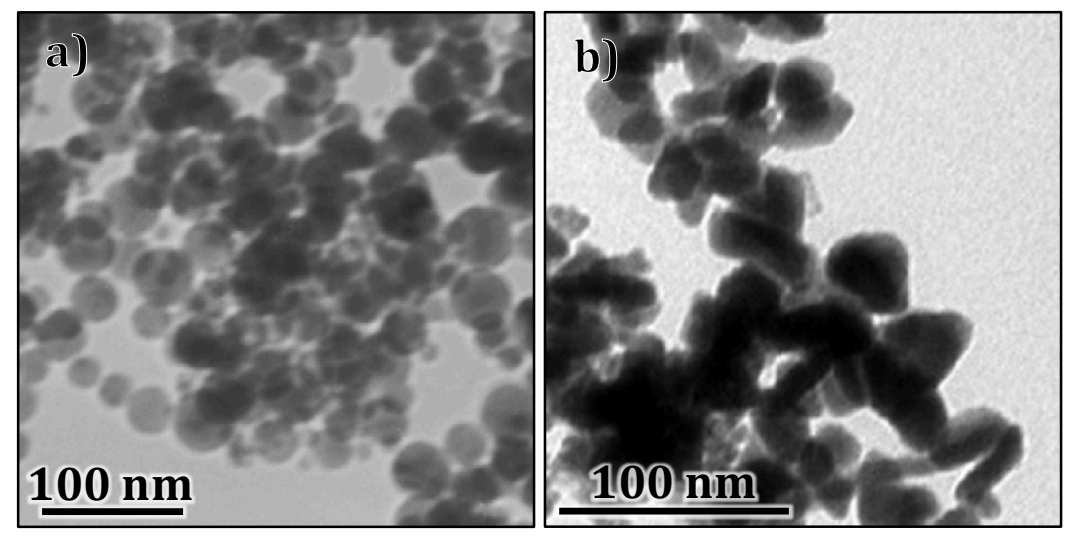

Figure 1.5: Bi NPs generated from aqueous protocols yielding hydrophobic particles with random morphologies and large size distributions. a) Bi NPs synthesized from $\mathrm{BiCl}_{3}$ using $\mathrm{N}_{2} \mathrm{H}_{4} \bullet \mathrm{H}_{2} \mathrm{O}$ as a reducing agent and SOA as a surface-stabilizing agent. Particles range in size from 25 - $40 \mathrm{~nm}$. Reproduced with permission from ref. 51, copyright 2008 Elsevier; b) Bi NPs synthesized from the chemical reduction of a bismuth tartrate precursor species with $\mathrm{NaH}_{2} \mathrm{PO}_{2}$, stabilizing the surface with SOA. Particles range in size from $10-50 \mathrm{~nm}$. Reproduced with permission from ref. 52, copyright 2011 Elsevier.

While these two synthetic protocols make steps toward a direct aqueous synthesis for Bi NPs, the particles that are generated are hydrophobic and must still 
undergo a ligand exchange in order to make them dispersible in aqueous solutions. To address this, there have been several syntheses developed in our lab whose aim was to directly synthesize aqueous, colloidally stable Bi NPs. The first was a pHdependent aqueous synthesis that used bismuth nitrate pentahydrate $\left(\mathrm{Bi}\left(\mathrm{NO}_{3}\right)_{3} \bullet 5 \mathrm{H}_{2} \mathrm{O}\right)$ as a solid suspension in a glycine buffer, titrated with $\mathrm{KOH}$ to reach various targeted $\mathrm{pH}$ solutions. ${ }^{53}$ Prior to reduction, an equilibrium between the free $\mathrm{Bi}^{3+}$ precursor and insoluble $\mathrm{Bi}(\mathrm{OH})_{3}$ was established. To initiate reduction and particle nucleation, sodium borohydride $\left(\mathrm{NaBH}_{4}\right)$ was added to the suspension after the addition of a solution of dextran, which served as the surface-stabilizing species. Experimentally, it was determined that the size distribution and morphology of the resulting particles relied heavily on the $\mathrm{pH}$ of the initial reaction solution. With increasing acidity, the solubility of $\mathrm{Bi}(\mathrm{OH})_{3}$ increases, increasing the availability of $\mathrm{Bi}^{3+}$ species that can be reduced to $\mathrm{Bi}^{0}$ monomer. This in turn affects the rate of Bi NP nucleation and growth, as a result of the increased number of available nuclei from which particles can grow. As seen in Figure 1.6a, for syntheses carried out in solutions with a $\mathrm{pH}<9$, the reactions are very rapid and the resulting particles are not uniform in size. However, for syntheses carried out in solutions with a $\mathrm{pH}>9$, the reaction kinetics become more controlled and an increase in size uniformity is observed. This can be attributed to the limited availability of the monomer in solution when the reducing agent is added to the system. With less $\mathrm{Bi}^{3+}$ available, a single nucleation event is observed, followed by subsequent diffusional growth from the remaining monomer. The most size uniform colloid resulted when the synthesis was conducted at a pH of 9.97, which produced Bi NPs that were 19.5 
$\pm 3.7 \mathrm{~nm}$ in diameter, as determined by transmission electron microscopy (TEM) size analysis (Figure 1.6b).
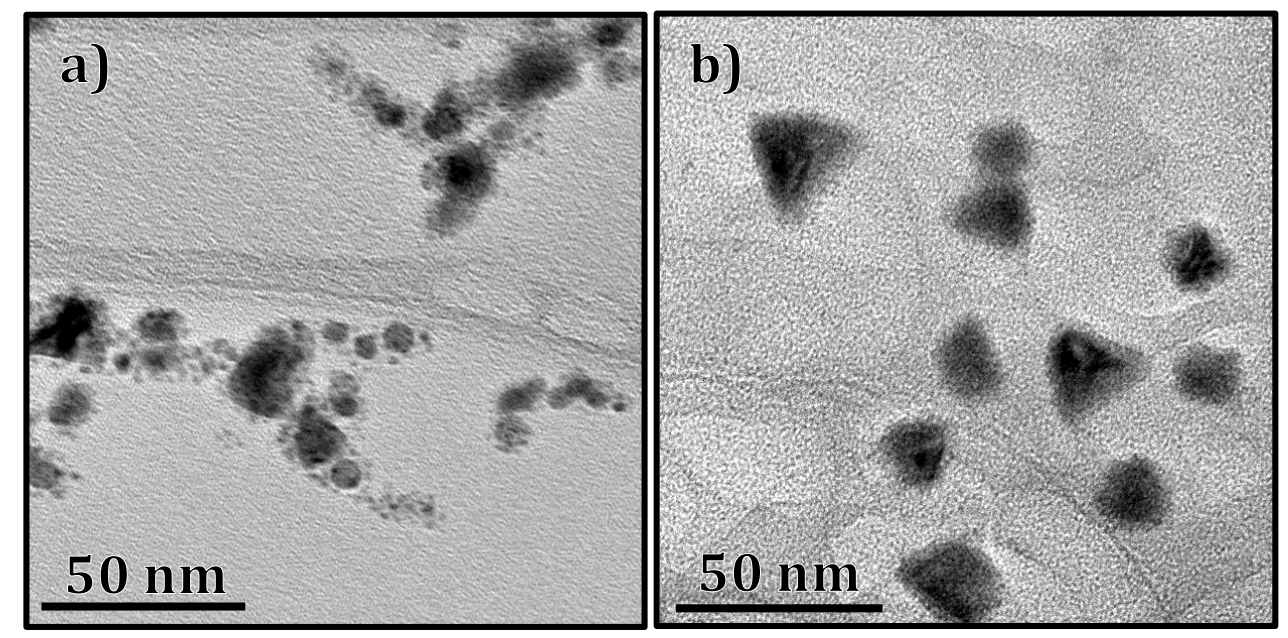

Figure 1.5: Bi NPs synthesized via an aqueous pH-dependent route. a) Particles synthesized below a $\mathrm{pH}$ of 9 , and; b) particles synthesized above a $\mathrm{pH}$ of 9 , demonstrating the dependence of size and shape on the $\mathrm{pH}$ of the reaction. Reproduced with permission from ref. 53, copyright 2012 American Chemical Society.

For this colloid, while the core size distribution was relatively small, the mean hydrodynamic diameter of the particles was measured to be $130 \mathrm{~nm}$ with a broad standard deviation. This size difference can be attributed to the polymeric surface-stabilizing agent and the hydration shell about each particle. Nonetheless, the Bi NPs generated from this protocol were colloidally and oxidatively stable in certain aqueous solutions, remaining stable for over one month when stored in solutions with a pH between 8 and 10, or in phosphate buffered saline solutions maintained at a $\mathrm{pH}$ of 7.4. These particles were prone to photodegradation, but remained robust when stored in the absence light over a wide range of temperatures, suggesting a good potential for biomedical use. 
Another protocol developed in our lab sought to generate aqueous colloids of Bi NPs, via chemical reduction in a polyol solution. ${ }^{8}$ In this reaction, $\mathrm{Bi}\left(\mathrm{NO}_{3}\right)_{3} \bullet 5 \mathrm{H}_{2} \mathrm{O}$ was dissolved in 1,2-propanediol (PPD), and combined with an extreme excess of $\alpha$ D-glucose, which acts not only as a surface stabilizing ligand, but is also a possible co-reductant in the reaction. Rapid reduction was initiated by the addition of borane morpholine and was allowed to proceed for one minute, at which point the reaction was quenched in electrophoretically pure water at $4^{\circ} \mathrm{C}$. The particles from this synthesis not only show great morphological uniformity, but are also relatively monodisperse in size, with particles from a best synthesis having a $74 \pm 14 \mathrm{~nm}$ core diameter, and an $86 \pm 35 \mathrm{~nm}$ hydrodynamic diameter, in which the crystalline $\mathrm{Bi}$ core constitutes $60 \%$ of the volume (Figure 1.7).

While yielding particles that are relatively uniform in size and shape, this particular synthesis generates a significant waste stream in order to isolate the Bi NP products from excess solvent and capping agent, which is standard in polyol syntheses, as mentioned earlier. In a standard reaction at a total volume of $\sim 100$ $\mathrm{mL}, 54 \mathrm{~g}$ of $\alpha$-D-glucose is used, making an almost 300:1 molar ratio of glucose-tobismuth. Furthermore, this reaction can generate up to $4 \mathrm{~L}$ of aqueous waste from the time of synthesis to the isolation of particles via centrifugation. Additionally, when particles are dialyzed for further purification, this generates another $1-2 \mathrm{~L}$ of aqueous waste. Therefore, while this synthesis represents the best Bi NP synthetic protocol for size and shape uniformity developed in our lab, the waste stream 
generated and the labor-intensive work up are issues that would be desirable to improve.

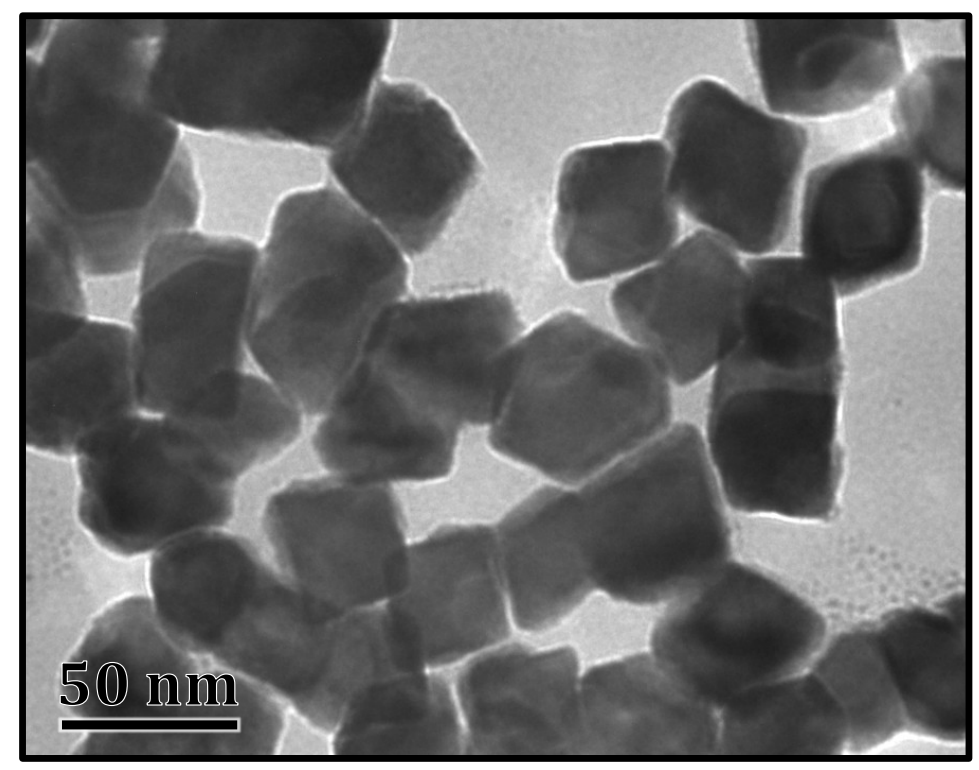

Figure 1.6: Bi NPs synthesized in PPD with the surface stabilizer and co-reductant $\alpha$-Dglucose. Reproduced with permission from ref. 8, copyright 2014 American Chemical Society.

\subsection{Conclusions}

As presented, there have been significant strides made in the development of aqueous protocols for the generation of Bi NPs. However, each presents its own particular issue, the most prevalent being the issue of solubility of bismuth (III) precursors in aqueous media. Our lab sought to circumvent this issue by moving to a polyol-based synthesis, which poses its own set of problems. While solubility is no longer at issue, the waste stream generated and laborious work up from these reactions is undesirable. The following research seeks to circumvent these issues by targeting a more atom economic, direct aqueous synthetic route, with equivalent or 
improved size uniformity, as well as tunability over a range of particle sizes, which is something our previous methods were not able to achieve. 


\section{Chapter 2 - Employing Redox Active Surface Stabilizing Agents in the Direct Synthesis of Colloidally Stable Aqueous Bismuth Nanoparticles}

\subsection{Abstract}

The initial aim of this work was to develop a synthesis for bismuth nanoparticles (Bi NPs) that takes advantage of the $\mathrm{pH}$-dependent isomeric equilibrium of glucose, which would allow for the reduction of bismuth (III) to elemental bismuth. The straight-chain aldehyde isomer of glucose was expected to serve as both the reducing agent and the surface-stabilizing agent. Due to the observed lack of control over morphology and size when glucose was used as a reducing agent, borane morpholine was added as a co-reductant to increase the rate of the reaction. Addition of the co-reductant was observed to result in better control over the size and morphology of the particles. However, the resulting NPs routinely underwent oxidative dissolution upon quenching the reaction, indicating ineffective surface stabilization on the part of glucose.

This prompted us to look at other avenues for stabilizing the particle surface. Consequently, we next employed $300 \mathrm{MW}$ poly(ethylene glycol) (PEG) in place of glucose, since the polymeric surface stabilizing agent was likely to afford a more stable particle surface. However, some syntheses yielded particles coated with PEG that were equally unstable post-synthesis, as evidenced by the oxidative dissolution of the nanoparticles upon quenching the reaction. Alternately, for some syntheses where immediate oxidative dissolution did not occur, the resulting particles were crystalline aggregate nanostructures with no uniformity. Interestingly, for the syntheses that resulted in immediate oxidative dissolution, when the reaction 
mixtures were left to sit in an airtight vessel in the dark for $\sim 96$ hours at room temperature, re-nucleation and growth of Bi NPs was observed. The Bi NPs synthesized in this way were both relatively size uniform and colloidally stable. In the majority of the above cases, the identity of the surface species could not be confirmed by FTIR or ${ }^{1} \mathrm{H}$ NMR, likely due to a lack of surface stabilizing agent on the NPs. This implies electrostatic stabilization of the Bi NP colloid.

\subsection{Introduction}

Elemental nanoparticles (NPs) have been of great interest in the past few decades, owing to their vast range of potential applications. From their size- and shape-dependent optical and magnetic properties to their ever-growing application as catalysts in organic synthetic transformations, ${ }^{1-3}$ elemental NPs continue to open up many new avenues of chemistry that were not yet accessible. One of the more exciting areas of research into elemental NPs lies within the field of biomedicalimaging, where nanoscale X-ray contrast agents (XCAs) present an attractive new class of contrast material owing to their potential for increased circulation times and the possibility of site directed imaging, both of which are limitations for the molecular, iodinated contrast agents that are currently in clinical use..$^{4-7}$

Elemental NPs based on elements such as gold, platinum, tantalum, and bismuth have been examined as possible NP XCAs in recent years. ${ }^{8-16}$ The choice of these elements in particular is due the fact that higher density materials and materials with higher atomic numbers are expected to exhibit enhanced attenuation of X-ray radiation relative to their molecular, iodinated counterparts. ${ }^{7}$ In addition to high density and high atomic number, NPs are comprised of a large number of 
attenuating atoms in a relatively small volume, which offers the possibility of enhanced localized contrast at lower effective clinical concentrations of contrast agent.

While bismuth NPs (Bi NPs) have so far demonstrated good catalytic ${ }^{17-19}$ and thermoelectric properties, ${ }^{20-22} \mathrm{Bi}$ NPs also have many attractive properties that may make them ideal XCAs in biomedical X-ray imaging applications, such as computed tomography (CT), relative to other elemental nanomaterials. ${ }^{23-30}$ This is a result of several different factors, the first being that the salt precursor species that are used to synthesize Bi NPs through either thermal decomposition or chemical reduction tend to be quite inexpensive (priced as low as $\$ 1 / \mathrm{g}$ ), ${ }^{31}$ owing to $\mathrm{Bi}$ being terrestrially more abundant ${ }^{32}$ than other high atomic number elements, such as gold or platinum. Additionally, a handful of studies have been conducted into the efficacy of Bi NPs as XCAs. One such study examined attenuation coefficients $(\mu$, as outlined in Chapter 1, Section 1.1.2) of likely NP contrast materials, such as bismuth $(Z=83)$, gold $(Z=79)$, platinum $(Z=78)$, tantalum $(Z=73)$, and iodine $(Z=53)[\mu(\mathrm{Bi})=5.74$ versus $\mu(\mathrm{Au})=5.16, \mu(\mathrm{Pt})=4.99, \mu(\mathrm{Ta})=4.30$, and $\mu(\mathrm{I})=1.94 \mathrm{~cm}^{2} \mathrm{~kg}^{-1}$ at $\left.100 \mathrm{kV}\right) \cdot{ }^{33}$ As reflected in the reported values of $\mu$ for each of the elements, Bi is expected to better attenuate X-ray radiation versus the other four elements.

Another study that was conducted in our lab measured attenuation as a function of effective concentration (i.e., rates of attenuation, in Hounsfield Units/mM) using common clinical X-ray tube voltages ( $80 \mathrm{kV}, 100 \mathrm{kV}, 120 \mathrm{kV}$, and $140 \mathrm{kV}$ ); the attenuation rates of Bi NPs were compared to both solvated Bi (III) cations and an iodinated standard. ${ }^{23}$ This study demonstrated that Bi outperforms 
the iodinated standard significantly at each of the four X-ray tube voltages examined. Furthermore, it was observed that as tube voltage increased, there was no statistically significant change in rate of attenuation for either Bi (III) cations or Bi NPs, unlike the iodinated standard. This indicates that Bi-based contrast agents should be widely applicable over a range of clinical conditions.

In addition to the ability of $\mathrm{Bi}$ to better attenuate $\mathrm{X}$-ray radiation and its low price point, there also lies a potential pathway to renal clearance for Bi NPs. Elemental $\mathrm{Bi}$ has been shown to undergo dissolution to some extent in oxidative environments, ${ }^{23}$ which suggests that Bi NPs would be expected to oxidatively decompose over time to give soluble Bi (III) species that could then be excreted from the body. Given the medicinal history of Bi (III) compounds over the centuries, ${ }^{34,35}$ this pathway to excretion is considered to be a biologically safe one. This is in stark contrast to elements such as gold or platinum that are oxidatively stable under physiological conditions, which inevitably leads to accumulation within the body and potential risk of toxicity. 36,37

Given the susceptibility of elemental Bi to oxidative decomposition, coupled with the relative instability of NPs in general, the synthesis of Bi NPs in aqueous media is challenging. To overcome this challenge, the majority of Bi NP synthetic literature focuses on bottom-up synthetic strategies that utilize hydrophobic organic solvents in air-free environments to mitigate oxidative dissolution; furthermore, many of these reactions require relatively high temperatures (e.g., greater than $\left.150^{\circ} \mathrm{C}\right) \cdot{ }^{38-45}$ One of the more notable Bi NP preparations in this category is the synthesis developed by F. Wang et al. that starts from a thermally unstable Bi 
precursor, $\mathrm{Bi}\left[\mathrm{N}\left(\mathrm{SiMe}_{3}\right)_{2}\right]_{3}$, and through a thermal decomposition reaction at $180^{\circ} \mathrm{C}$, generates highly uniform, polymer-coated Bi NPs that are size tunable through the subtle alteration of synthetic conditions. ${ }^{42}$ However, the polymer used in the synthesis, poly(1-vinylpyrrolidone)-graft-(1-hexadecene), results in Bi NPs that are hydrophobic and would need to undergo an exchange of the surface stabilizing agent in order for the resulting particles be biomedically useful. For this purpose, it would be desirable to develop a more direct approach to synthesize aqueous Bi NPs, while still retaining the attractive size control and size tunability achieved in organic phase syntheses. Currently, there exist only a handful of direct aqueous syntheses for Bi NPs, 18,19,46-48 several of which ultimately generate hydrophobic particles due to stabilization of the particle surfaces with amphiphilic surfactants, such as sodium oleate.

Our lab has sought to develop a method that produces stable, aqueous Bi NPs through either a direct aqueous route, or through a non-aqueous approach that results in hydrophilic particles in a single synthetic step. We previously reported a direct aqueous route that utilizes a pH-dependent solubility equilibrium of Bi (III) nitrate in equilibrium with free Bi (III) ions, which are reduced using sodium borohydride $\left(\mathrm{NaBH}_{4}\right)$ and stabilized with dextran in a glycine-buffered solution. ${ }^{48}$ The kinetics of this reaction are highly dependent on the availability of free $\mathrm{Bi}^{3+}$ ions, which is reliant on the solution $\mathrm{pH}$. This synthetic method yielded hydrophilic particles in a single reaction step, which were relatively uniform in size, but not in shape. Furthermore, for typical products of this synthetic method, the Bi core constituted only $4 \%$ of the total particle volume ( $96 \%$ is polymeric shell), which 
led to difficulty in concentrating the particles enough to produce solutions with strong X-ray contrast.

To gain better control over the reaction kinetics, thereby providing improved particle size and shape uniformity, an ideal Bi (III) precursor should be fully soluble in the reaction medium. Therefore, an indirect route to hydrophilic Bi NPs was developed using 1,2-propanediol (PPD) as the solvent of synthesis, which after workup, ultimately resulted in aqueous, colloidal Bi NPs. ${ }^{8}$ In this reaction, PPD was used to solubilize Bi (III) nitrate, presumably as a coordination complex of $\mathrm{Bi}^{3+}$ and the vicinal diol of PPD, and glucose was used in place of dextran polymer to try to reduce the ratio of organic shell to Bi core. Because the reaction was accomplished at relatively low temperatures, a chemical reducing agent was added (i.e., borane morpholine, which is considered kinetically slow in comparison to $\left.\mathrm{NaBH}_{4}\right)^{49} v s$. relying on thermally initiated reduction (i.e., as is done in polyol synthesis of metallic NPs, see Chapter 1 , Section 1.3.1). The products of this synthetic method resulted in better uniformity in shape and size versus the prior, direct aqueous route, as well as achieved a more ideal ratio of poorly attenuating surfactant to highly attenuating Bi NP core ( $\sim 64 \%$ of the total particle volume). This resulted in a higher X-ray attenuation rate compared to iodinated contrast agents, as described previously in this section.

While this non-aqueous synthetic approach offered better kinetic control, yielding relatively uniform and stable aqueous Bi NPs, the overall method from reaction to particle isolation has a few challenges, the most prevalent being the extensive waste stream generated. Typically, in a single reaction, $54 \mathrm{~g}$ of glucose are 
used, which corresponds to an approximately 300:1 molar ratio of glucose-to-Bi. Additionally, in a typical workup, several liters of aqueous waste are generated in the removal of solvent and reaction byproducts, which is accomplished via centrifugation and dialysis. To address these challenges, a method that is performed entirely in water would be beneficial, but should begin with a fully soluble precursor to avoid the polydispersity challenges encountered in our first aqueous preparation method. Therefore, in this chapter, we report the development of a direct aqueous synthesis of Bi NPs using a soluble Bi (III) tartrate precursor.

\subsection{Experimental}

\subsubsection{Materials}

Bismuth (III) nitrate pentahydrate was purchased from Acros Organics at a purity of 98\%. $\alpha$-D-glucose ((glc), anhydrous, 96\%), D-(-)-tartaric acid (D-TA, 99\%) and polyethylene glycol ((PEG), $1000 \mathrm{MW}$ ) were purchased from Aldrich. Borane morpholine complex $(B M)$ was purchased from Alfa Aesar at a purity of 97\%. PEG 300 was purchased from TCI and PEG 3350 was purchased from Sigma. Certified ACS Plus Grade concentrated nitric acid $\left(\mathrm{HNO}_{3}\right)$ was purchased from Fisher Scientific. Sodium hydroxide $(\mathrm{NaOH})$ was purchased from Fisher Scientific at a purity of $\geq 97.0 \%$. All chemicals were used as received without any further purification.

\subsubsection{Synthesis}

\subsection{2a - Aqueous Precursor Preparation}


To prepare the Bi (III) tartrate precursor, $625 \mu \mathrm{L}$ of a $0.5 \mathrm{M} \mathrm{Bi}$ (III) nitrate pentahydrate solution in $1.908 \mathrm{M} \mathrm{HNO}_{3}$ (aqueous) was added dropwise to $10 \mathrm{~mL}$ of a $50 \mathrm{mM}$ D-TA solution in a $100 \mathrm{~mL}$ round bottom flask at room temperature while continuously stirring at $550 \mathrm{rpm}$. The addition of the aqueous Bi (III) solution to the D-TA solution immediately resulted in a white precipitate, which is presumed to be a Bi (III) tartrate coordination complex. This mixture was then titrated with $\sim 1.5 \mathrm{M}$ $\mathrm{NaOH}$ to dissolve the white precipitate.

2.3.2b - Synthesis of Bismuth Nanoparticles Using Glucose as Reductant

For each synthesis carried out, the Bi (III) precursor was prepared as described above in Section 2.3.2a. The solution $\mathrm{pH}$ of the dissolved Bi (III) tartrate precursor was further adjusted with $\sim 1.5 \mathrm{M} \mathrm{NaOH}$ to reach various target $\mathrm{pH}$ values, ranging from $8-13$. The $\mathrm{pH}$-adjusted Bi (III) precursor solutions were then diluted to a volume of $55 \mathrm{~mL}$ using pre-made $\mathrm{NaOH}$ solutions at $\mathrm{pH} 8,9,10,11,12$, and 13 . The solutions were then heated to reach a reaction temperature of $80^{\circ} \mathrm{C}$, and held at this temperature for a period of 30 minutes prior to addition of the reductant. Then, $\alpha$-D-glucose $(1-6$ molar equivalents, relative to $\mathrm{Bi})$, dissolved in a corresponding $\mathrm{pH} 8$ - 13 solution, was added to the reaction mixtures to achieve total volumes of $60 \mathrm{~mL}$. The addition of glucose solution was observed to initiate the reactions, as evidenced by the appearance of dark black colloids. Immediately after observation of color change, the reactions were quenched by pouring the contents of the $100 \mathrm{~mL}$ round bottom flasks into $250 \mathrm{~mL}$ beakers containing $100 \mathrm{~mL}$ of DI $\mathrm{H}_{2} \mathrm{O}$ at $\sim 0^{\circ} \mathrm{C}$. Quenched reactions were then evenly distributed between four $50 \mathrm{~mL}$ 
centrifuge tubes and centrifuged for 30 minutes at $4.4 \mathrm{krpm}$. Finally, the supernatants were decanted and the particles were re-dispersed in DI $\mathrm{H}_{2} \mathrm{O}$, sonicated, and centrifuged two additional times. The obtained particles were ultimately re-dispersed and stored in DI water.

2.3.2c - Synthesis of Bismuth Nanoparticles Using Borane Morpholine as CoReductant

For each synthesis carried out, the Bi (III) precursor was prepared as described above in Section 2.3.2a. The solution $\mathrm{pH}$ was further adjusted to achieve an approximate value of 12 with the addition of $\sim 1.5 \mathrm{M} \mathrm{NaOH}$. The dissolved Bi (III) precursor solution was first diluted with DI water to a volume of $50 \mathrm{~mL}$ and then heated to reach a reaction temperature of $80^{\circ} \mathrm{C}$. After holding at the reaction temperature for 30 minutes, 3 molar equivalents of $\alpha$-D-glucose (relative to $\mathrm{Bi}$ ), dissolved in $5 \mathrm{~mL}$ of $\mathrm{DI}_{2} \mathrm{O}$, was added. Borane morpholine was then dissolved in 5 $\mathrm{mL}$ DI $\mathrm{H}_{2} \mathrm{O}$, at molar equivalents ranging from $0.5-3$ (relative to $\mathrm{Bi}$ ). The borane morpholine solutions were added to the dissolved Bi (III) precursor solutions immediately following the addition of $\alpha$-D-glucose. Each reaction had a total volume of $60 \mathrm{~mL}$ and was initiated upon the addition of borane morpholine. The reactions were quenched and the resulting Bi NPs were isolated as detailed in Section 2.3.2b.

\subsection{2d - Synthesis of Bismuth Nanoparticles Using PEG as Surface Capping Agent}

For each synthesis carried out, the Bi (III) precursor was prepared as described above in Section 2.3.2a. The solution $\mathrm{pH}$ was further adjusted to achieve an approximate value of 10 with the addition of $\sim 1.5 \mathrm{M} \mathrm{NaOH}$. The dissolved Bi (III) 
precursor solution was first diluted with DI water to a volume of $50 \mathrm{~mL}$ and then heated to reach a reaction temperature of $80^{\circ} \mathrm{C}$. After holding for a period of 30 minutes at the reaction temperature, 1, 3, 8.5, 17, or 25.5 molar equivalents of PEG 300 (relative to $\mathrm{Bi}$ ) was then dissolved in the reaction solution and $\mathrm{DI}_{2} \mathrm{H}_{2} \mathrm{O}$ was added to adjust the volume to $55 \mathrm{~mL}$. Additional reactions were also done in an identical manner, only using PEG 1000 or PEG 3350 in place of PEG 300, but with no significant change in resulting NP sizes or morphologies. Borane morpholine at 0.5 molar equivalents (relative to $\mathrm{Bi}$ ) was then dissolved in $5 \mathrm{~mL}$ of $\mathrm{DI}_{2} \mathrm{O}$ and added to the dissolved Bi (III) precursor solution. Each reaction had a total volume of $60 \mathrm{~mL}$ and was initiated upon the addition of borane morpholine. The reactions were quenched and the resulting Bi NPs were isolated as detailed in Section 2.3.2b.

2.3.2e - Incubation Reactions Producing Bi Nanoparticles with PEG as Surface Stabilizer

Upon quenching Bi NPs generated from the syntheses using PEG as a surfacestabilizing agent, most samples would be observed to undergo dissolution, as evidenced by loss of the black solution color. These samples were then left in sealed centrifuge tubes and kept in the dark at room temperature for a period of $\sim 96 \mathrm{~h}$, during which time the black solution color re-appeared. The resulting Bi NPs were then isolated as detailed in Section 2.3.2b.

\subsubsection{Bismuth Nanoparticle Characterization}

pH Measurements

Measurements of $\mathrm{pH}$ were accomplished using an Accumet Basic ab15 pH meter calibrated with standard buffer singlet-solutions obtained from VWR at pH values of 
4.01, 7.01, and 10.01. The $\mathrm{pH}$ was measured in each reaction solution just prior to the addition of an aqueous $\alpha$-D-glucose solution.

Dynamic Light Scattering (DLS)

DLS measurements were taken on a Horiba LB-550 dynamic light scattering instrument, and an instrumental algorithm was used to supply the hydrodynamic diameter distribution. Samples were prepared by concentrating nanoparticle colloids via rotary-evaporation. The concentrated particle solutions were then transferred to a standard $1 \mathrm{~cm}$ path length quartz cuvette in $\mathrm{DI} \mathrm{H}_{2} \mathrm{O}$ filtered through a $450 \mathrm{~nm}$ PTFE syringe filter. Measurements (1000 scans) at varying concentrations of each sample were performed in order to determine a size distribution independent of concentration effects.

Transmission Electron Microscopy (TEM)

TEM was performed on an FEI Tecnai F-20 TEM operating at $200 \mathrm{kV}$. Isolated particles were re-suspended in dry methanol and drop cast onto holey carbon $\mathrm{Cu}$ supported TEM grids and allowed to dry at room temperature for at least 1 hour prior to imaging. Lattice fringe spacing and particle sizes were determined using ImageJ $1.49 \mathrm{v}$ software analysis.

X-ray Diffraction (XRD)

Bi NPs were pressed onto a glass support slide, and XRD data were collected in focused beam (Bragg-Brentano) geometry on a Rigaku Ultima IV X-ray diffraction system using graphite monochromatized $\mathrm{Cu} \mathrm{K} \alpha$ radiation. Scans were performed over the angular range $20-70^{\circ} 2 \theta$ at a scan rate of $0.2^{\circ} / \mathrm{min}$ at room temperature. 
Fourier Transform Infrared Spectroscopy (FTIR)

FTIR measurements were performed on a Thermo Scientific Nicolet iS10 FTIR spectrophotometer equipped with a single-bounce diamond attenuated total reflectance (ATR) attachment. Spectra were processed using OMNIC analytical software. Samples were prepared by concentrating the reaction mixtures via rotaryevaporation, removing reaction solvent and any remaining volatile organics prior to measurement.

Proton Nuclear Magnetic Resonance Spectroscopy $\left({ }^{1} H\right.$ NMR)

${ }^{1} \mathrm{H}$ NMR spectroscopy was performed on a Bruker $400 \mathrm{MHz}$ AVANCE II+ NMR spectrometer using a standard pulse sequence (Zg30) and Bruker automation software. Spectra were processed using the MestReNova 9 analysis software package. Samples were prepared by drying the particles via rotary-evaporation after washing with dry methanol. Once isolated, particles were dissolved, depending on solubility, in $\sim 50-75 \mu \mathrm{L}$ of $\mathrm{CDCl}_{3}$, DMSO-d6, or $\mathrm{D}_{2} \mathrm{O}$ and were then transferred to a borosilicate NMR tube with a micropipette. The samples were then diluted to final volumes of $\sim 500-600 \mu \mathrm{L}$ with the appropriate deuterated solvent.

\subsection{Results and Discussion}

To establish an appropriate reaction temperature for the aqueous reduction of the Bi-tartrate precursor, all reaction sets were initially screened over a temperature range from room temperature $(\mathrm{rt})$ to $100^{\circ} \mathrm{C}$, where the upper limit was dictated by the boiling point of water. In general, reactions carried out between rt and $60^{\circ} \mathrm{C}$ were observed to be kinetically slow, which would be expected to lead to Bi(III) reduction and particle nucleation occurring over a long period of time. 
Conversely, when the reaction temperature was $100^{\circ} \mathrm{C}$, reaction rates were observed to be too rapid, which often led to large, aggregated masses of bismuth. Therefore, we elected to use $80^{\circ} \mathrm{C}$ as the standard reaction temperature, since the intermediate reaction kinetics would be most likely to result in uniform nucleation and growth of colloidally stable (i.e., not aggregated) Bi NPs.

2.4.1 pH-Dependent Synthesis of Bismuth Nanoparticles Using Glucose as a Reductant

As previously described in Section 2.2, our lab developed a synthetic approach that produced stable hydrophilic Bi NPs after workup by using Bi (III) nitrate pentahydrate as the Bi (III) precursor, PPD as the reaction solvent, $\alpha$-Dglucose as the surface-stabilizing agent, and borane morpholine as the chemical reducing agent. While it was initially believed that glucose only surface-passivated the particles, it was later discovered that glucose also affected the reaction kinetics, i.e., the addition of glucose decreased the reaction time from approximately one hour to one minute, as compared to reduction with borane morpholine alone. ${ }^{50}$ This result suggested that glucose may either serve as a catalyst for the borane morpholine reaction with Bi (III), or directly reduce the Bi (III), or both. Thus, we attempted to employ glucose as the sole, stoichiometric reducing agent in a direct aqueous synthesis of Bi NPs, which if successful, would minimize purification efforts in removing non-aqueous solvent (PPD) and large excesses of glucose that were drawbacks of the prior method. 
It has been well documented that reducing sugars, such as glucose, are capable of reducing cationic metal species to their elemental state. ${ }^{51}$ In aqueous media, glucose is known to establish an equilibrium having three dominant isomers: the alpha and beta cyclic forms, and the ring-opened aldehyde form (Scheme 2.1a), which can serve as a reductant. ${ }^{52}$ The straight-chain aldehyde form of D-glucose is known to be oxidized in the presence of excess base, to result in a $2 \mathrm{e}^{-}$transfer (Scheme 2.1b) to a suitable oxidizing agent, such as a metal cation..$^{51}$ Oxidation of the aldehyde results in lowering the concentration of the ring-opened form in the glucose isomerization equilibrium, which is expected to shift in response to generate more ring-opened D-glucose, as dictated by Le Châtelier's principle; thus, a steady supply of the redox active aldehyde is expected to be maintained. Taking advantage of this expectation, reducing sugars have previously been utilized as reducing agents in syntheses of $\mathrm{Ag}, \mathrm{Au}, \mathrm{Pt}, \mathrm{Pd}$, and $\mathrm{Cu}$ NPs, where the sugar oxidation product may also be the surface stabilizing species. ${ }^{53-55}$
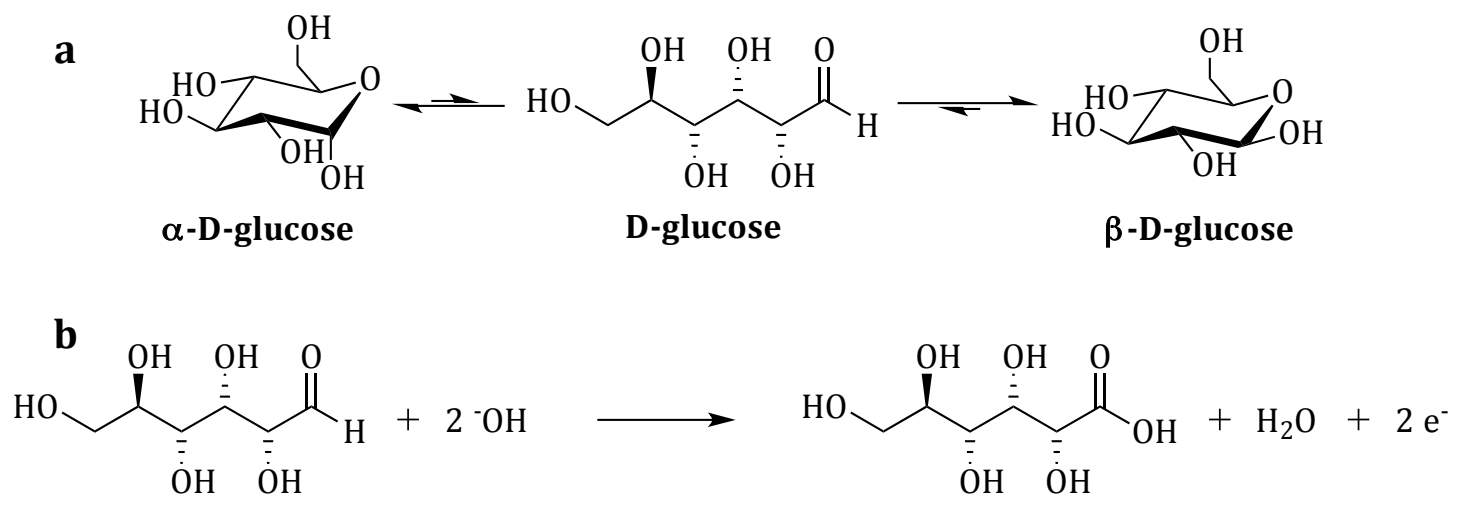

Scheme 2.1: (a) Isomeric aqueous equilibrium of glucose, where the $\alpha$ and $\beta$ isomers are the dominant species, and the straight chain, redox-active isomer makes up less than $1 \%$ of 
the isomeric equilibrium. (b) Redox half-reaction of ring-opened D-glucose in alkaline media, where oxidation of one glucose molecule yields a two-electron transfer.

To generate a water-soluble Bi (III) precursor in high $\mathrm{pH}$ aqueous solutions, where glucose oxidation is favored, the inclusion of a coordinating ligand, such as D(-)-tartaric acid (D-TA) was necessary, since common Bi (III) salts are water soluble only at low pH. At low pH, elemental $\mathrm{Bi}$, once formed, would be expected to dissolve readily. Additionally, when the Bi (III) cation is un-complexed and in the presence of $-\mathrm{OH}$ ions, an equilibrium between hydrated $\mathrm{Bi}(\mathrm{III})$ cations and insoluble $\mathrm{Bi}(\mathrm{OH})_{3}$ is established (as described in Chapter 1, Section 1.3.2), where $\mathrm{Bi}(\mathrm{OH})_{3}$ will eventually undergo a dehydration reaction to form the more thermodynamically stable $\mathrm{Bi}_{2} \mathrm{O}_{3}$ as a light-yellow precipitate. ${ }^{23}$ However, D-TA forms a complex with Bi (III), initially generating an insoluble white precipitate that can be subsequently dissolved by the addition of $\mathrm{NaOH}$ (Scheme 2.2). This complex is of unknown stoichiometry (Bi $\mathrm{B}_{\mathrm{x}}(\mathrm{D}-$ TA)y and charge, but it is stable toward hydrolysis, as well as highly water soluble over a wide $\mathrm{pH}$ range (from $\mathrm{pH} \sim 5.50$ to $\mathrm{pH} \sim 14$ ).

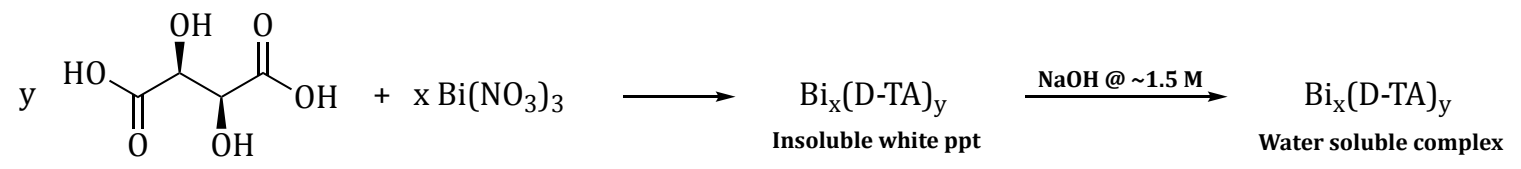

Scheme 2.2: Scheme depicting the reaction of D-(-)-tartaric acid with Bi (III) to generate an insoluble $\mathrm{Bi}$ (III) tartrate precipitate. The precipitate is titrated with $\mathrm{NaOH}$ to achieve solubility of the precursor species.

Initial studies were carried out using 3 molar equivalents of glucose (relative to $\mathrm{Bi}$ ). It was observed over a series of screening reactions, where $1-6$ molar 
equivalents of glucose were used, that the rate of reaction wasn't greatly affected with the addition of more or less glucose. In order to ascertain the effect of $\mathrm{pH}$ on the reduction of the $\left(\mathrm{Bi}_{x}(\mathrm{D}-\mathrm{TA})_{\mathrm{y}}\right)$ complex by glucose, reactions were carried out in solutions with different $\mathrm{pH}$ values, ranging from 8 to 13 , and the reduction of the $\left(\mathrm{Bi}_{\mathrm{x}}(\mathrm{D}-\mathrm{TA})_{\mathrm{y}}\right)$ complex by glucose was observed to be highly $\mathrm{pH}$-dependent. For example, when syntheses were carried out at a pH between 8 and 10, no reaction took place, as evidenced by the observation that these reaction solutions remained colorless over a $24 \mathrm{~h}$ period. However, when the $\mathrm{pH}$ was in the range of $11-12$, Bi (III) reduction was observed to take place over a period of 30 minutes, as evidenced by the darkening of the reaction solution to an eventual black colloid, indicative of elemental Bi on the nanoscale.

TEM imaging and analysis on Bi NPs synthesized from solutions having $\mathrm{pH}$ in the range of $11-12$ showed no shape or size uniformity (Figure 2.1a \& b). The resulting particles appeared to be large aggregates, where each aggregate is composed of smaller particles. Based on the black solution color, these aggregates were assumed to be elemental $\mathrm{Bi}$, which was confirmed with high-resolution TEM (HRTEM); HRTEM showed a lattice spacing of $3.15 \AA$, corresponding to the (012) plane of elemental Bi (Figure 2.1c). ${ }^{48}$ In solution, these aggregates were shown to have a bimodal distribution with an average hydrodynamic diameter (HD) of 163.3 $\pm 143.5 \mathrm{~nm}$, via DLS analysis (Figure 2.1d). Taking a closer look at the DLS distribution, the mean diameter of the smaller population (with the highest frequency) is approximately $150 \mathrm{~nm}$, while many large aggregates with a mean diameter around $1.15 \mu \mathrm{m}$ were also observed. 
Lack of uniformity in the size and shape of the resultant Bi NPs may have resulted from a number of factors, including the possibility that precursor reduction, and subsequent nanoparticle nucleation and growth, take place over too broad of a time period (i.e. 30 minutes, vs. a more ideal burst nucleation). This would be expected to result in the growth of existing nuclei into nanoparticles as new nuclei are still forming, to cause a broad size distribution. Secondly, the tendency of the resulting Bi NPs toward aggregation suggests that there is insufficient surface passivation, which may result in oxidation of the particle surfaces by water.
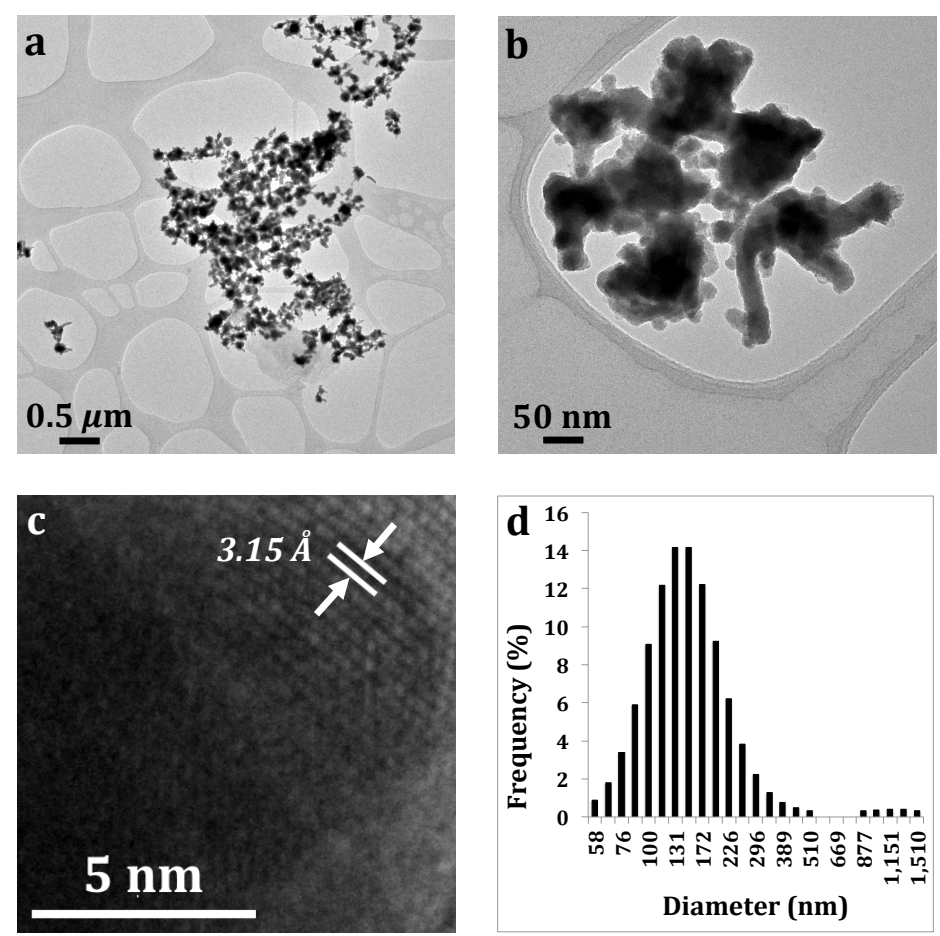

Figure 2.1: a) and b) TEM images of resulting Bi NPs synthesized by reduction of the $\mathrm{Bi}_{\mathrm{x}}(\mathrm{D}-$ TA)y precursor using D-glucose as a reducing agent in basic media; c) HRTEM of Bi NP lattice fringes measured at $3.15 \AA$ corresponding to the (012) plane of elemental bismuth; 
d) DLS histogram of resulting Bi NPs showing a bimodal distribution with an average HD of $163.3 \mathrm{~nm} \pm 143.5 \mathrm{~nm}$.

Surface analysis with FTIR showed broad absorption features over two different regions, ranging from $4000-2150 \mathrm{~cm}^{-1}$ and $2000-650 \mathrm{~cm}^{-1}$. In the first region, no distinct functionality can be ascertained, as there is no clear definition to the absorbance features. The second region, however, has discernable absorption bands at $1720 \mathrm{~cm}^{-1}, 1535 \mathrm{~cm}^{-1}, 1308 \mathrm{~cm}^{-1}$, and $1035 \mathrm{~cm}^{-1}$ within the broadband absorbance, perhaps indicating the presence of a carboxylate group (SI Figure S1). However, the presence of glucose or gluconic acid could not be confirmed. ${ }^{1} \mathrm{H}$ NMR spectra showed only signals for water and the deuterated solvent being used.

Versus the glucose reduction of the $\mathrm{Bi}_{\mathrm{x}}(\mathrm{D}-\mathrm{TA})_{\mathrm{y}}$ precursor in aqueous solutions with $\mathrm{pH}=11-12$, reactions with $\mathrm{pH}=13$ resulted in faster kinetics, where reduction and nanoparticle formation took place in a slightly decreased time period. However, the resulting nanoparticles synthesized at $\mathrm{pH} 13$ showed no distinct differences from those synthesized in solution with $\mathrm{pH}$ in the range of $11-12$. Additionally, as a consequence of the highly alkalinity, the Bi (III) precursor would often times remain oxidized and would hydrolyze to result in highly uniform, spherical $\mathrm{Bi}_{2} \mathrm{O}_{3}$ NPs (Figure 2.2a \& b). A relatively small particle size and narrow size distribution was routinely observed for the $\mathrm{Bi}_{2} \mathrm{O}_{3}$ NPs, with a representative sample synthesized at $\mathrm{pH}=13$ having an average diameter of $8.54 \mathrm{~nm} \pm 1.43 \mathrm{~nm}$, as measured through TEM analysis (Figure 2.2c). PXRD analysis confirmed the identity of the NPs as that of the $\alpha$-phase of $\mathrm{Bi}_{2} \mathrm{O}_{3}$ (Figure 2.2d), which was further confirmed 
with HRTEM, where a measured lattice spacing of $4.02 \AA$ corresponds to the (020) plane of $\alpha-\mathrm{Bi}_{2} \mathrm{O}_{3}$ (Figure 2.2e). ${ }^{56}$

While this approach proved successful in reducing Bi (III) to elemental Bi NPs when the solution pH was between 8 and 12, it was considered not viable for the production of stable, aqueous Bi NP colloids that would be suitable for use in biomedicine. The lack of size and shape uniformity in the resulting Bi NPs suggests that the reaction kinetics fall outside of ideal conditions for uniform NP formation. Additionally, the observed aggregation of synthesized particles suggests poor surface stabilization by glucose. Furthermore, the high alkalinity needed to promote glucose oxidation presents its own issues, since at sufficiently high $\mathrm{pH}, \mathrm{Bi}$ (III) is prone to hydrolysis.
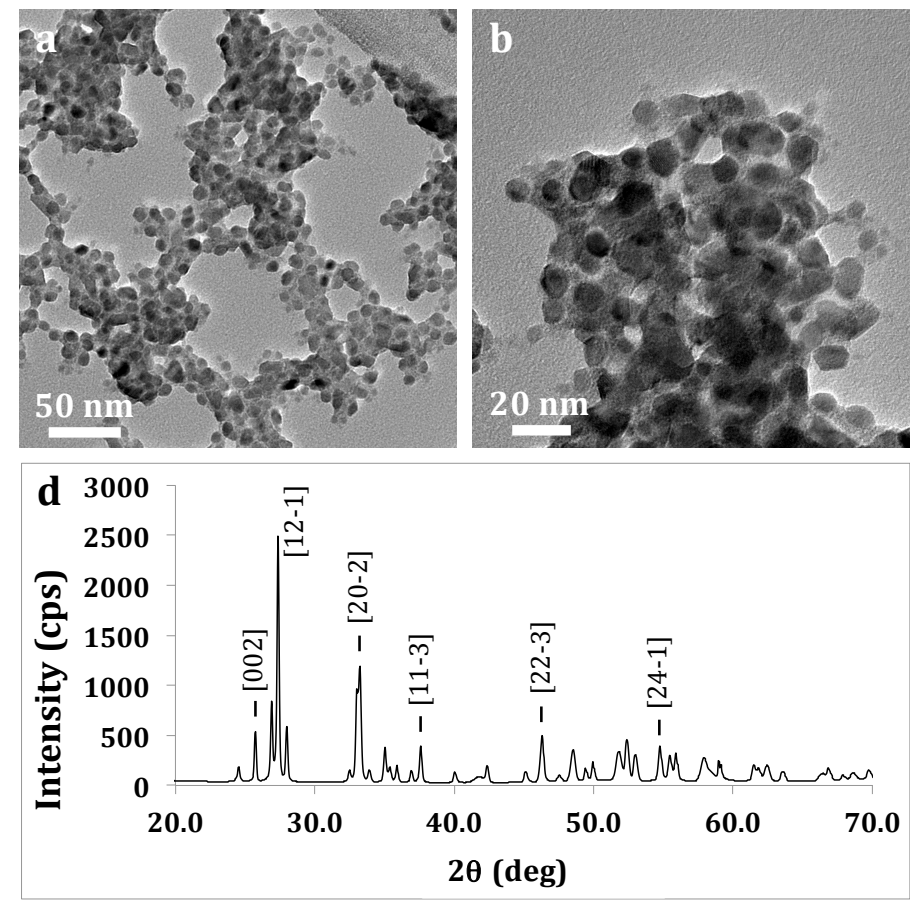
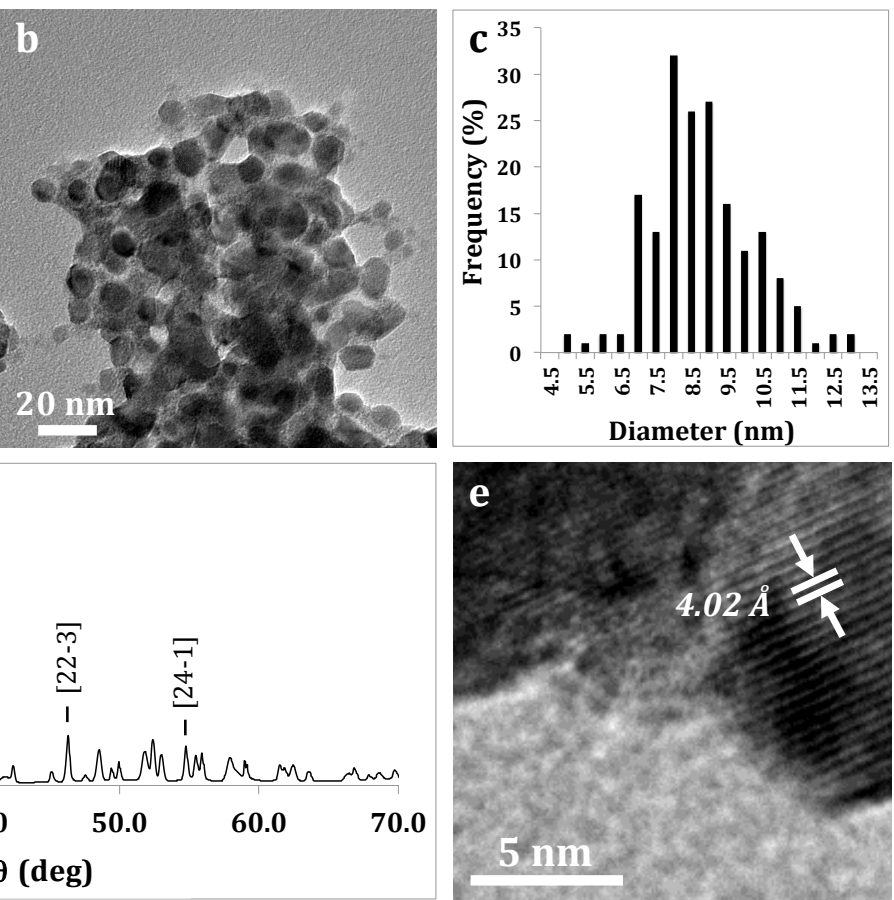

Figure 2.2: a) and b) TEM of $\mathrm{Bi}_{2} \mathrm{O}_{3}$ NPs formed through hydrolysis of the Bi tartrate precursor as the result of the highly alkaline synthetic environment; c) histogram of the 
particle size distribution as measured through TEM analysis; d) PXRD of isolated NPs indexed as $\alpha-\mathrm{Bi}_{2} \mathrm{O}_{3}$; e) HRTEM showing lattice fringes with spacing measured at $4.02 \AA$, indicative of $\alpha-\mathrm{Bi}_{2} \mathrm{O}_{3}$.

\subsubsection{Borane Morpholine-Assisted Synthesis of Bismuth Nanoparticles}

In an effort to address the poor reaction kinetics observed using glucose as the sole reducing agent, a co-reductant was added to the reaction system. In doing so, it was hypothesized that the nucleation and growth stages could be better separated to facilitate the formation of more discrete and uniform nanoparticles. Given that borane morpholine (BM) had been used successfully in the glucoseassisted polyol synthesis developed in our lab, this seemed to be a suitable choice for a co-reductant species.

To ascertain the effect of the added reducing agent, molar equivalence trials were performed, where a range of 0.5 - 3 molar equivalents of BM were used to initiate $\mathrm{Bi}$ (III) reduction. Syntheses were carried out at a solution $\mathrm{pH}$ of approximately 12 , where Bi (III) hydrolysis was less likely and the best reactivity in the glucose studies was observed. As expected, the addition of BM significantly increased the reaction rate. With the addition of 3 equivalents of $\mathrm{BM}$, reaction times were observed to be near instantaneous, resulting in uncontrolled particle formation and growth, where the particles immediately aggregated and crashed out of solution. Decreasing the quantity of added BM resulted in better control over the synthetic system, as reaction times increased with decreasing amounts of BM. The 
best control was achieved with the addition of 0.5 molar equivalents of BM, where reactions were carried out for $\sim 30 \mathrm{~s}$ and subsequently quenched.

As can be seen in Figure 2.3a \& b, the addition of 0.5 molar equivalents of BM successfully promoted the formation of discrete particles. HRTEM shows a lattice spacing of $3.18 \AA$ (Figure 2.3c), corresponding to the (012) plane of elemental Bi. However, the particles varied in size significantly over replicate syntheses, with average hydrodynamic diameters ranging from $32.5 \mathrm{~nm}$ to $172.9 \mathrm{~nm}$ by DLS analysis (Figure 2.3d \& e). Furthermore, the size distributions were observed to be broad, e.g., $\sim 38 \%$ for the two products shown in Figure $2.3 \mathrm{~d} \&$ e.
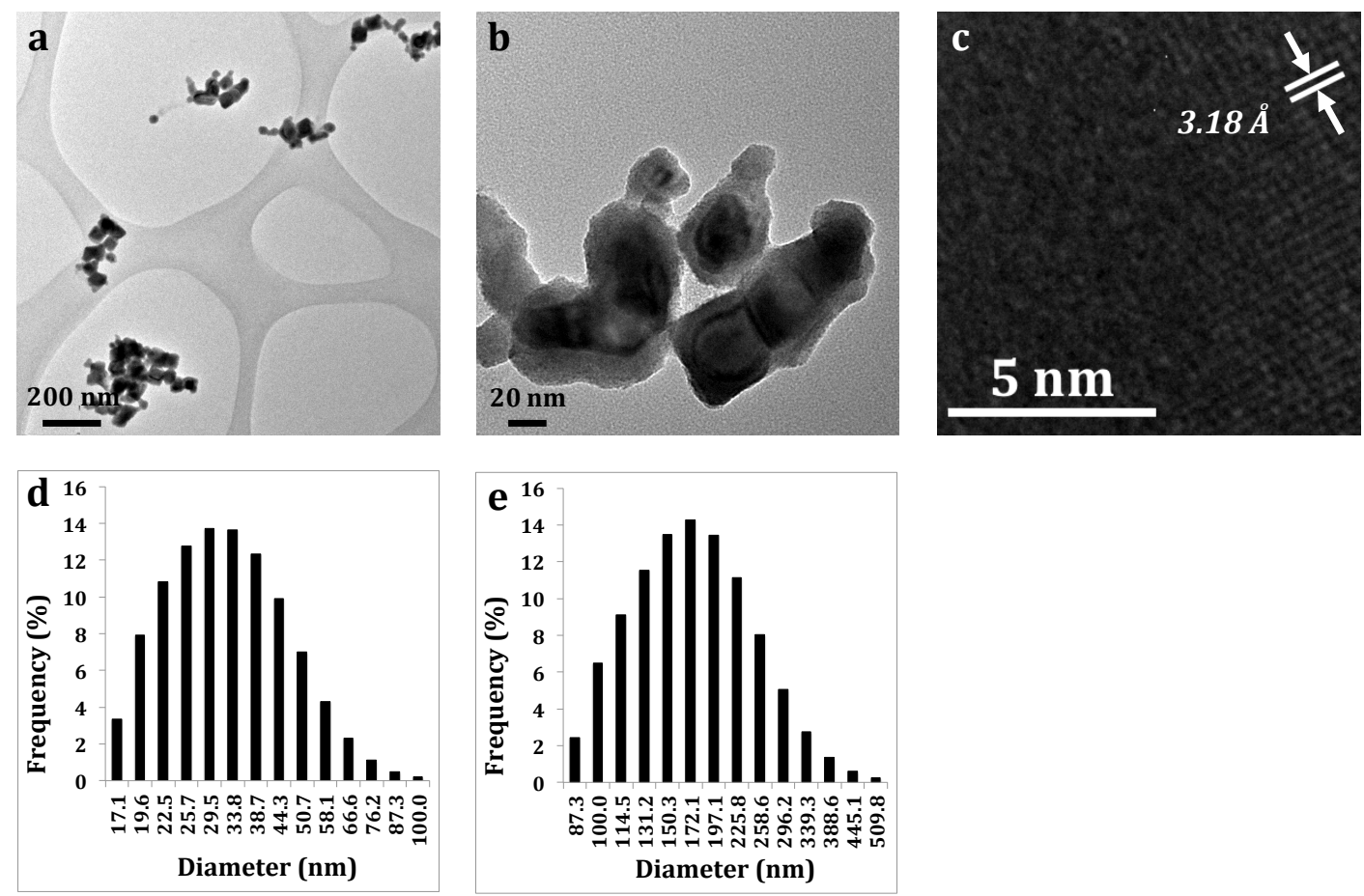

Figure 2.3: a) and b) TEM images of Bi NPs synthesized with the addition of borane morpholine as a co-reductant to glucose; c) HRTEM of resulting Bi NPs showing a lattice spacing of $3.18 \AA$, indicative of the (012) planes of elemental Bi; d) and e) DLS histograms of 
resulting Bi NPs showing inconsistent size distributions when reactions are quenched after the same time interval - d) $32.5 \mathrm{~nm} \pm 12.4 \mathrm{~nm}$ and e) $172.9 \mathrm{~nm} \pm 64.9 \mathrm{~nm}$.

The variation in size can most likely be attributed to the rapid rate of particle growth, coupled to the imprecise nature of determining the reaction end point. With the introduction of a co-reductant, the rate of $\mathrm{Bi}$ (III) reduction was effectively increased, which was observed to result in a more rapid particle nucleation event. However, an increase in the rate of particle growth is also likely under these conditions, which is supported by the immediate precipitation of large, aggregated particles when 3 equivalents of BM were used. Since the reaction end point is determined observationally by the appearance of a black solution color, slight variations in reaction duration could account for the different sizes because of the fast growth rate. As a further complication, if particle growth is not allowed to proceed for a long enough duration prior to quenching the reaction, the particles that are formed undergo oxidative dissolution, as evidenced by the disappearance of the black solution color.

The lack of stability of these colloids towards dissolution, which is similar to the Bi NPs synthesized without the addition of BM, suggests that glucose is not effectively passivating the NP surface, allowing for oxidative dissolution to occur. Indeed, neither glucose nor gluconic acid were detected by FTIR or ${ }^{1} \mathrm{H}$ NMR. The FTIR contained only broad, un-assignable features, and the ${ }^{1} \mathrm{H}$ NMR spectra obtained were characteristic of solvent, similar to those described in Section 2.4.1 
where glucose was used as the sole reducing agent. Thus, no definitive determination of the surface species was made.

2.4.3 Synthesis of Bismuth Nanoparticles Using PEG 300 as a Surface-Stabilizing Agent

In both of the above cases, no evidence was obtained to indicate that glucose (or gluconic acid) effectively passivated the particle surfaces. Thus, surface instability of the resulting Bi NPs was a suspected cause of particle aggregation and oxidative dissolution. To overcome this issue, investigation into a different surfacestabilizing agent was necessary. The use of poly(ethylene glycol) (PEG) as a nanoparticle surface-stabilizing agent in biomedical applications, such as drugdelivery and imaging, is very well studied. ${ }^{57}$ PEG is often used to coat the surface of nanoparticles because of its ability to avoid recognition by the reticuloendothelial system (RES), ultimately resulting in prolonged circulation times. Additionally, given the hydrophilicity of the repeating ethylene ether units of the polymer and the terminal hydroxyl groups, solubility in aqueous media tends to be very high. Thus, it was reasoned that polymeric PEG coatings would both impart water solubility to the Bi NPs, as well as improve the oxidative stability by better passivating the particle surfaces (vs. a small molecule surfactant, such as glucose).

Initial trials were carried out using PEG 300 at 1 and 3 molar equivalents of the polymer relative to bismuth. For these syntheses, the solution $\mathrm{pH}$ was maintained at a value of approximately 10 . Since glucose had been removed as a reducing species, an elevated solution $\mathrm{pH}$ was no longer a necessity. In general, while black aqueous colloids were routinely obtained, the particles obtained from 
these syntheses exhibited poor size and shape uniformity, similar to the nanoparticles obtained through the glucose-reduction route. In the TEM images, the particles were observed to be large crystalline aggregates (Figure $2.4 \mathrm{a} \& \mathrm{~b}$ ), and HRTEM measurement of the lattice spacing provided confirmatory evidence that these particles were elemental $\mathrm{Bi}$, with a lattice spacing measured at $3.15 \AA$, corresponding to the (012) plane of elemental Bi (Figure 2.4c). Analysis via DLS gave an average hydrodynamic diameter of $86.7 \mathrm{~nm} \pm 37.6 \mathrm{~nm}$ (Figure $2.4 \mathrm{~d}$ ).

By removing glucose from the reaction system, it is believed that the kinetic advantage gained by the addition of borane morpholine to the glucose reduction system was lost. The removal of glucose effectively slowed the rate of Bi (III) reduction, likely resulting in a prolonged period of nucleation, similar to what was observed in the glucose reduction route. Additionally, the morphology of the resulting particles suggests that PEG did not effectively passivate the NP surfaces, leading to the degree of aggregation observed. 

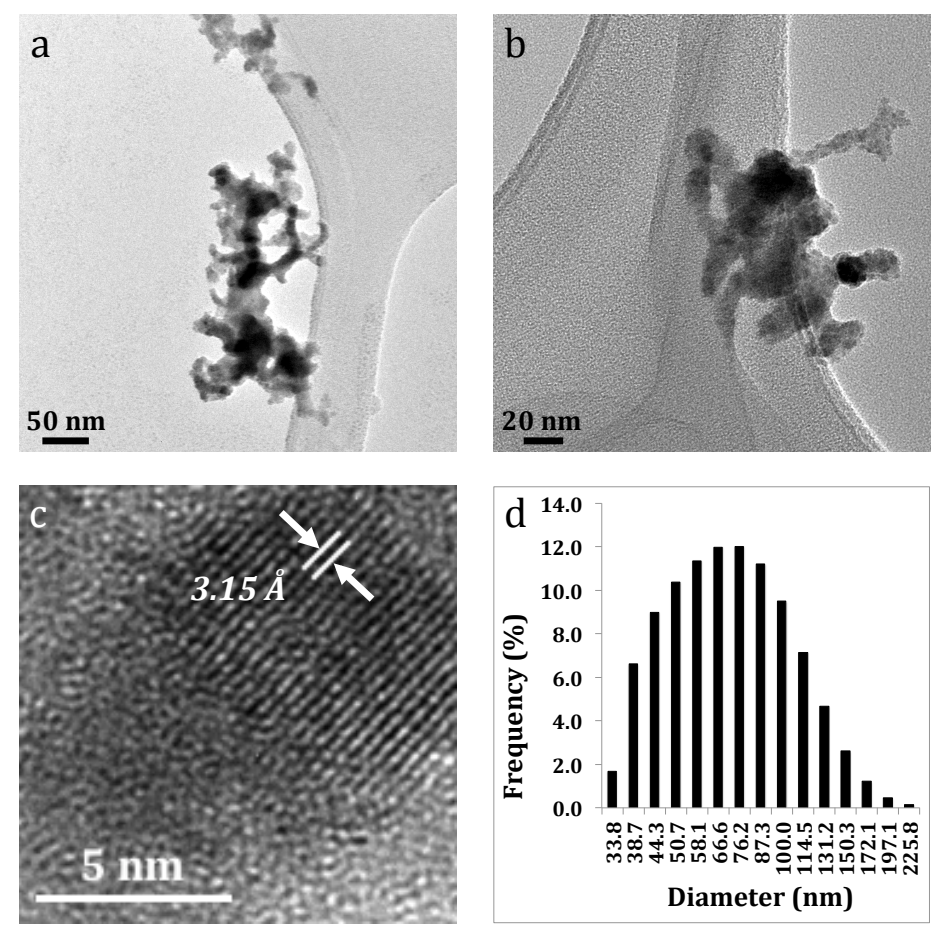

Figure 2.4: a) \& b) TEM images of Bi NPs synthesized using PEG 300 as a surface-stabilizing agent and borane morpholine as the sole reducing agent; c) HRTEM of Bi lattice fringes measured at $3.15 \AA$ A corresponding to the (012) plane; d) DLS histogram of resulting Bi NPs showing an average HD of $86.7 \mathrm{~nm} \pm 37.6 \mathrm{~nm}$.

FTIR analysis of the particles may suggest the presence of some PEG 300 (SI Figure S2) on the NP surfaces. A weak, broad absorption band from $3500 \mathrm{~cm}^{-1}$ to $3050 \mathrm{~cm}^{-1}$ was observed, in the region characteristic of a hydrogen-bonded $-\mathrm{OH}$ stretch; two sharp $-\mathrm{CH}_{2}$ stretching bands at $2922 \mathrm{~cm}^{-1}$ and $2852 \mathrm{~cm}^{-1}$ could definitively be assigned. Absorption bands are also observed at $1446 \mathrm{~cm}^{-1}$ and 1367 $\mathrm{cm}^{-1}$, which are consistent with the reference spectrum for PEG 300. However, there are additional bands observed at $1734 \mathrm{~cm}^{-1}$ and $1558 \mathrm{~cm}^{-1}$, indicating the presence of a carboxylic acid/carboxylate group. It's unclear if these absorbance bands are a result of tartrate surface functionalization, or if we may possibly have a 
carboxylated PEG species. Unfortunately, characterization via ${ }^{1} \mathrm{H}$ NMR could not further elucidate the nature of the surface species, where spectra obtained were characteristic of solvent, similar to those described in Section 2.4.1

To try to mitigate the issues of poor morphology and aggregation, additional reactions were carried out using 8.5, 17, and 25.5 molar equivalents of PEG 300, relative to bismuth. PEG 300 was also replaced in another series of reactions using larger PEG species (PEG 1000 and PEG 3350) having higher molecular weights. In these additional reactions, no significant changes in resulting NP sizes or morphologies were observed. Additionally, regardless of the relative amount of PEG, or the PEG molecular weight, the Bi NPs synthesized through this route were prone to oxidative dissolution, particularly if particle growth was not allowed to proceed for a long enough duration.

Unexpectedly, for batches that underwent immediate oxidative dissolution upon quenching of the reaction, when left in an airtight vessel in the dark for $\sim 96 \mathrm{~h}$ at room temperature, particle regrowth appeared to take place, as evidenced by the reappearance of a black solution color. However, for a parallel sample that was left to stand in ambient light, no particle regrowth was observed. Previous studies have shown elemental Bi NPs to be prone to photodegradation, whereupon exposure to ambient light, Bi NP colloids were observed to decompose over a period of 14 days. ${ }^{48}$ This supports the lack of particle regrowth of the dissolved colloid when exposed to ambient light. 

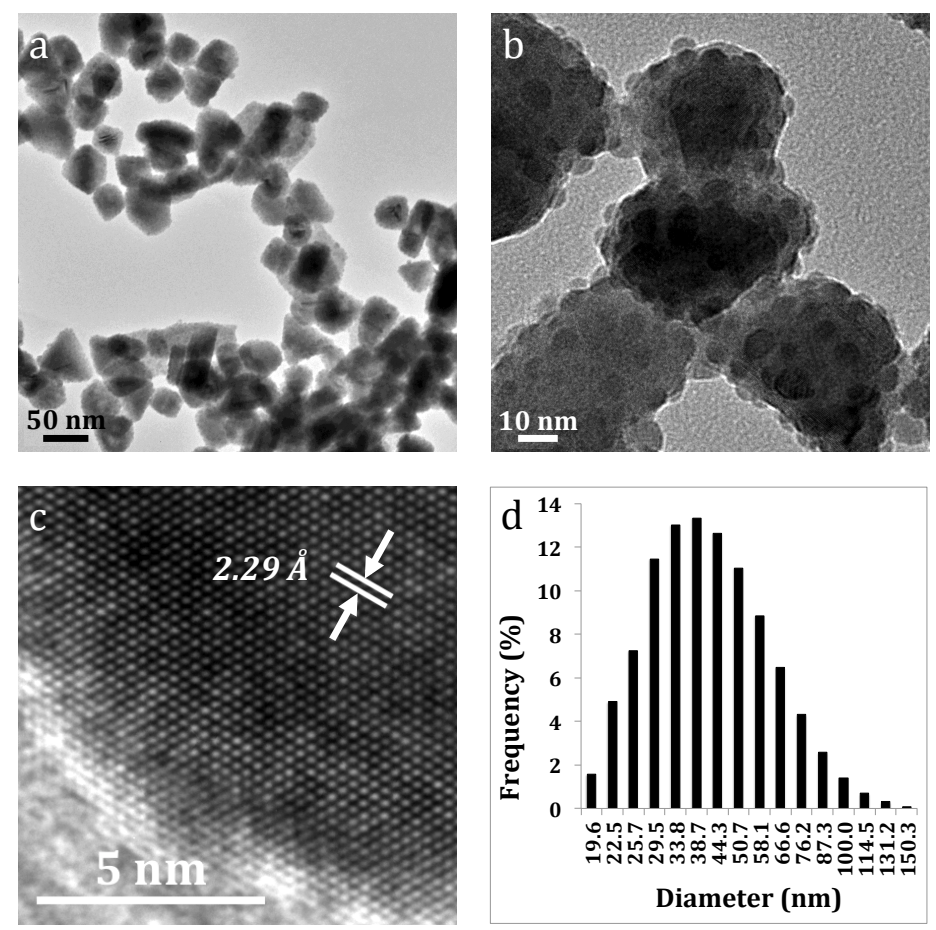

Figure 2.5: a) \& b) TEM images of Bi NPs synthesized through incubation of dissolved Bi NP batches that used PEG 300 as the surface-stabilizing agent and borane morpholine as the reducing agent; c) HRTEM of Bi lattice fringes measured at $2.29 \AA ̊$ corresponding to the (110) plane of elemental Bi; d) DLS histogram of resulting Bi NPs showing an average HD of $37.7 \mathrm{~nm} \pm 16.3 \mathrm{~nm}$.

Surprisingly, the Bi NPs obtained through this "incubation" route exhibited much greater size and shape uniformity (Figure $2.5 \mathrm{a} \& \mathrm{~b}$ ) vs. all of the aqueous syntheses described above. In the TEM images, each particle appears to be composed of a discrete Bi core, which is covered by smaller, highly uniform $\sim 5 \mathrm{~nm}$ Bi particles. Further, the HRTEM image and analysis revealed a lattice spacing of $2.29 \AA$, which corresponds to the (110) plane of elemental Bi (Figure 2.5c). ${ }^{24}$ Through DLS analysis, it was determined that the particles synthesized through this 
incubation route show a somewhat uniform size distribution, with an average hydrodynamic diameter of $37.7 \mathrm{~nm} \pm 16.3 \mathrm{~nm}$ (Figure $2.5 \mathrm{~d}$ ).

Surface characterization, via FTIR, of the Bi NP colloid produced similar results to those observed in Section 2.4.1 (SI Figure S3). The spectrum shows a broadband absorbance from $4000 \mathrm{~cm}^{-1}$ to about $2200 \mathrm{~cm}^{-1}$. There are several distinct absorption bands at $1539 \mathrm{~cm}^{-1}, 1302 \mathrm{~cm}^{-1}$, and $1027 \mathrm{~cm}^{-1}$. These absorbance features might indicate the presence of a carboxylate group, but could not be confirmed, as spectra obtained via ${ }^{1} \mathrm{H}$ NMR were characteristic of solvent.

The Bi NPs generated from this "incubation" route were thought to be generated through a seed-mediated particle growth mechanism, which may explain the seed over-coated core morphology observed. Upon oxidative dissolution, it was hypothesized that not all the Bi particles had been totally dissolved. Instead, it is possible that some small Bi nuclei remained, which could then re-nucleate the dissolved Bi to enable particle regrowth. This process of digestive particle ripening, could be expected to result in the increased size and shape uniformity observed. This hypothesis was examined by a series of control reactions, in which all reactants were combined under the same conditions as the heated bench-top reaction, with the exception of applying heat to the system. The reactions were then placed in the dark at room temperature for a period of 96 hours.

These control reactions did not yield similar results. While Bi reduction was observed to take place, as evidenced by the evolution of a black solution color, the resulting Bi structures obtained were morphologically dissimilar to the products produced when the reaction is initially heated. Bi NPs and wires were routinely 
obtained from these control reactions, as well as large Bi microstructures and spherical amorphous particles (Figure 2.6a, b, \& c), where the size distributions measured via DLS are also dissimilar to those observed for the heated bench-top incubation reactions (Figure 2.6d). The results of these controls suggest that the initial heated bench-top reaction is necessary for the generation of nuclei, which then act as seeds for which controlled particle regrowth may then occur.
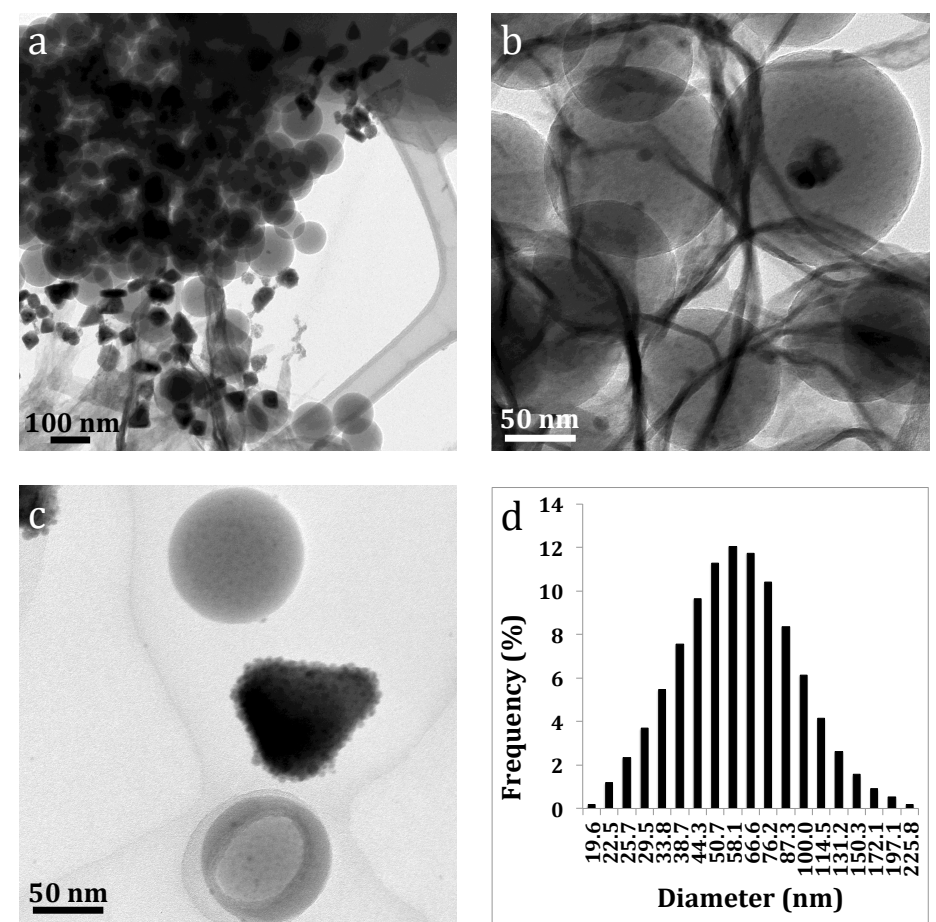

Figure 2.6: a), b) \& c) TEM images from several control reactions in which the soluble $\mathrm{Bi}$ (III) precursor, PEG 300, and borane morpholine are combined and left to incubate in the dark at room temperature for a period of $\sim 96 \mathrm{~h}$ resulting in Bi NPs, nanowires, and amorphous polymeric spheres; d) DLS histogram showing a large size distribution, with an average HD of $62.2 \mathrm{~nm} \pm 29.4 \mathrm{~nm}$. 


\subsection{Conclusions}

In each of the initial approaches described above, with the exception of the PEG 300 incubation route, the visual determination of the reaction endpoint was a predominant source of inconsistency in the obtained results, due to the incomplete separation of nucleation and growth periods, as well as a high rate of particle growth. In general, modifications made to affect the size, morphology and colloidal stability of particles generated from each route, such as changing $\mathrm{pH}$, exchanging a molecular for a polymeric surface stabilizer, or increasing surface-stabilizing agent concentrations, were ineffective. Similarly, increasing the polymer length of PEG also had no effect on the size or morphology of the resulting particles.

It was also found that following workup and isolation of the Bi NP colloids, the intended surface species were not observed through FTIR and ${ }^{1} \mathrm{H}$ NMR analysis. This may be the result of one of several factors. It's possible that the intended surface species (glucose or PEG 300) may have been stripped from the particle surface during workup either by intensive heating or vacuum during rotary evaporation to remove water. On the other hand, this may be due to electrostatic stabilization of the particles as a result of surface oxide formation. Unfortunately, this point remains unclear.

However, the PEG 300 incubation route was successful as a direct aqueous synthesis of colloidally stable, aqueous Bi NPs using inexpensive, biologically compatible reagents. Through an initial heated bench top reaction, initially formed Bi NP colloids underwent oxidative dissolution. However, upon dissolution, Bi nuclei remained in solution, which acted as seeds to promote slow diffusional growth of Bi 
NPs that show improved uniformity, with an average hydrodynamic diameter of $37.7 \mathrm{~nm} \pm 16.3 \mathrm{~nm}$. 


\section{Chapter 3 - One-Step Synthesis of Tartrate-Stabilized Aqueous Colloidal \\ Bismuth Nanoparticles}

\subsection{Abstract}

We report the direct aqueous synthesis of colloidal Bi NPs through a simple chemical reduction method using a soluble bismuth (III) tartrate precursor. Through this route, particles are easily isolated and re-dispersed in water by adjusting $\mathrm{pH}$, which cuts synthetic waste-streams dramatically and moves toward a more green approach versus traditional synthetic routes. Particles were synthesized in the presence of hexamethylenediamine (HMD), which was intended to act as the surface-stabilizing agent. However, characterization of the particle surface through FTIR analysis determined that it was the tartrate ligand of the bismuth (III) precursor that covers the particle surface.

\subsection{Introduction}

Bismuth nanoparticles (Bi NPs) have been the focus of research for a number of applications, from heterogeneous catalysis ${ }^{1-3}$ to thermoelectric applications. ${ }^{4-6}$ In particular, Bi NPs have been recently investigated in the area of biomedical X-ray attenuation as a new class of contrast material, ${ }^{7-14}$ owing to the high atomic number and density of bismuth, ${ }^{15}$ as well as relative biological tolerance to $\mathrm{Bi}$ compounds. ${ }^{16,17}$ To be an effective contrast material for biological use, such particles must be water-soluble. However, the development of a facile method for the preparation of water soluble, amino- or carboxylic-acid functionalized Bi NPs is a useful, yet challenging synthetic goal. Bismuth (III) salts tend to suffer from poor solubility in both polar and apolar solvents and will preferentially form insoluble 
precipitates with chalcogen species, such as oxygen, sulfur, and selenium, ${ }^{18}$ making bismuth (III) precursor selection a difficult task. In water, soluble bismuth (III) solutions are typically only achievable under highly acidic conditions, ${ }^{16}$ unless complexed with a water-soluble ligand. For example, Xia et al. demonstrated a direct aqueous synthetic route that uses an ammonium bismuth citrate $(\mathrm{ABC})$ complex as a water-soluble bismuth (III) precursor, which is chemically reduced with sodium borohydride $\left(\mathrm{NaBH}_{4}\right)$ in the presence of a soluble starch surface-stabilizing agent to generate colloidal aqueous Bi NPs. ${ }^{19}$ However, the particles synthesized show little size or shape uniformity as a result of the sparing solubility of the $\mathrm{ABC}$ complex, as well as from the oxidative instability of elemental bismuth in water, which is a common shortcoming with direct aqueous Bi NP syntheses.

To bypass the challenges of direct aqueous synthesis, there have been many alternative methods developed with which to synthesize Bi NPs. Thermal decomposition of bismuth precursors, such as bismuth (III) trimethylsilylamide $\left(\mathrm{Bi}\left[\mathrm{N}\left(\mathrm{SiMe}_{3}\right)_{2}\right]_{3}\right),{ }^{20-22}$ bismuth (III) alkylamides $\left(\mathrm{Bi}\left(\mathrm{NR}_{2}\right)_{3}\right),{ }^{23}$ or bismuth (III) thiolates $\left(\mathrm{Bi}(\mathrm{SR})_{3}\right)^{24,25}$ has, by and large, shown the best resulting product. Nanoparticles prepared through these routes typically show exceptional size and shape uniformity, and average sizes can often be tuned, while maintaining narrow size distributions, with minor changes to synthetic conditions. However, these nanoparticles are typically only soluble in nonpolar solvents prior to additional surface modification, which greatly inhibits their use for biological application.

Herein, we report a simple, aqueous synthetic strategy for Bi NPs using low cost reagents under mild synthetic conditions. Bi NPs were synthesized in the 
presence of hexamethylenediamine (HMD), which was intended to serve as a surface stabilizing ligand that could provide aqueous solubility through the polar, water soluble terminal amines, while providing oxidative stability with a hydrophobic barrier provided by methylene linkages between the terminal amines. The resultant Bi NPs produced from this synthesis were found to be highly stable when stored in water for up to several months. Additionally, nanoparticle isolation was achieved simply by centrifugation and re-dispersion of the flocculate by adjusting the $\mathrm{pH}$.

\subsection{Experimental}

\subsubsection{Materials}

Bismuth (III) nitrate pentahydrate was purchased from Acros Organics at a purity of 98\%. D-(-)-tartaric acid (D-TA) was purchased from Aldrich at a purity of $99 \%$. Borane morpholine complex $(B M)$ was purchased from Alfa Aesar at a purity of 97\%. Hexamethylenediamine (HMD) was purchased from Acros Organics at a purity of $99.5+\%$. Certified ACS Plus Grade concentrated nitric acid $\left(\mathrm{HNO}_{3}\right)$ was purchased from Fisher Scientific. Sodium hydroxide $(\mathrm{NaOH})$ was purchased from Fisher Scientific at a purity of $\geq 97.0 \%$. All chemicals were used as received without any further purification.

\subsubsection{Synthesis}

The insoluble $\mathrm{Bi}_{\mathrm{x}}(\mathrm{D}-\mathrm{TA})_{\mathrm{y}}$ precursor was prepared as outlined in Chapter 2 Section 2.3.2a. To solubilize the precursor, $2.2 \mathrm{~mL}$ of $\sim 1.5 \mathrm{M} \mathrm{NaOH}$ was added dropwise to dissolve the white precipitate. HMD at 1 molar equivalent relative to bismuth was then added to the precursor solution, which was subsequently diluted 
with DI water to achieve a volume of $50 \mathrm{~mL}$. The round bottom flask was then submerged in a temperature controlled oil bath set to a temperature of $60^{\circ} \mathrm{C}$ and allowed to reach reaction temperature for an interval of 30 minutes. Borane morpholine, at 0.5 molar equivalents relative to bismuth, was then dissolved in 10 mL of DI $\mathrm{H}_{2} \mathrm{O}$ just prior to addition. Once borane morpholine was added to the bismuth-containing solution, the reaction was allowed to proceed for approximately 30 minutes, at which time the reaction was quenched in $100 \mathrm{~mL}$ DI water at $\sim 0^{\circ} \mathrm{C}$. The reaction was replicated several times using these conditions. To the quenched reactions, $16 \mathrm{~mL}$ of $\mathrm{HCl}$ at $0.1 \mathrm{M}$ was added dropwise, causing the formed particles to flocculate and fall out of solution. The supernatant was then decanted, and the resulting solution was diluted to a volume of $100 \mathrm{~mL}$ with $\mathrm{DI} \mathrm{H}_{2} \mathrm{O}$ and evenly distributed between two $50 \mathrm{~mL}$ centrifuge tubes. These samples were centrifuged for 1 minute at $4.4 \mathrm{krpm}$. The supernatants were again decanted and the particles were washed and centrifuged one additional time with $20 \mathrm{~mL} \mathrm{DI} \mathrm{H}_{2} \mathrm{O}$. Finally, the supernatants were once more decanted, and the particles were re-dispersed by adding $\sim 1.5 \mathrm{M} \mathrm{NaOH}$ dropwise to the flocculate until a black colloidal dispersion of Bi NPs was observed.

\subsubsection{Bismuth Nanoparticle Characterization}

\section{Dynamic Light Scattering (DLS)}

DLS measurements were taken on a Horiba LB-550 dynamic light scattering instrument, and an instrumental algorithm was used to supply the hydrodynamic diameter distribution. Samples were prepared by concentrating nanoparticle colloids via rotary-evaporation. The concentrated particle solutions were then 
transferred to a standard $1 \mathrm{~cm}$ path length quartz cuvette in $\mathrm{DI} \mathrm{H}_{2} \mathrm{O}$ that had first been filtered through a $450 \mathrm{~nm}$ PTFE syringe filter. Measurements (1000 scans) at varying concentrations of each sample were performed in order to determine a size distribution independent of concentration effects.

Transmission Electron Microscopy (TEM)

TEM was performed on an FEI Tecnai F-20 TEM operating at $200 \mathrm{kV}$. Isolated particles were re-suspended in dry methanol and dropcast onto holey carbon $\mathrm{Cu}$ supported TEM grids and allowed to dry at room temperature for at least $1 \mathrm{hr}$ prior to imaging. Lattice fringe spacing and particle sizes were determined using ImageJ $1.49 \mathrm{v}$ software analysis.

$X$-ray Diffraction $(X R D)$

Bi NPs were pressed onto a glass support slide, and XRD data were collected in focused beam (Bragg-Brentano) geometry on a Rigaku Ultima IV X-ray diffraction system using graphite monochromatized $\mathrm{Cu} \mathrm{K} \alpha$ radiation. Scans were performed over the angular range $20-70^{\circ} 2 \theta$ at a scan rate of $0.2^{\circ} / \mathrm{min}$ at room temperature. Fourier Transform Infrared Spectroscopy (FTIR)

FTIR measurements were performed on a Thermo Scientific Nicolet iS10 FTIR spectrophotometer equipped with a single-bounce diamond ATR attachment. Samples were prepared by concentrating the reaction mixture via rotaryevaporation, removing reaction solvent and any remaining volatile organics prior to measurement.

Proton Nuclear Magnetic Resonance Spectroscopy $\left({ }^{1} H\right.$ NMR) 
${ }^{1} \mathrm{H}$ NMR spectroscopy was performed on a Bruker $400 \mathrm{MHz}$ AVANCE II+ NMR spectrometer using a standard pulse sequence (Zg30) and Bruker automation software. Spectra were processed using the MestReNova 9 analysis software package. Samples were prepared by first concentrating the reaction mixture via rotary-evaporation. Upon concentration, depending on solubility, $\sim 50-75 \mu \mathrm{L}$ of sample were transferred to a borosilicate NMR tube with a micropipette and filled to a final volume of $\sim 500-600 \mu \mathrm{L}$ with $\mathrm{CDCl}_{3}$, DMSO-d6, or $\mathrm{D}_{2} \mathrm{O}$.

\subsection{Results and Discussion}

To synthesize colloidally stable Bi NPs, a Bi $(\mathrm{D}-\mathrm{TA})_{\mathrm{y}}$ precursor was dissolved in an aqueous solution of the surfactant, hexamethylenediamine (HMD), and brought to a reaction temperature of $80^{\circ} \mathrm{C}$. Following the addition of the reducing agent, borane morpholine, a stable black colloid of crystalline Bi NPs was obtained. The morphology of Bi NPs synthesized in the presence of HMD was monitored using transmission electron microscopy (TEM), where various nanoparticle morphologies were observed. The majority of the resulting particles were observed to be ellipsoidal in shape; however, nanorods and plates were also routinely obtained (Figures $3.1 \mathrm{a} \& \mathrm{~b}$ ). Measurements via DLS showed an average hydrodynamic diameter of $33.4 \pm 12.5 \mathrm{~nm}$, indicating a good degree of size-uniformity (Figure 3.1c). Particle crystallinity was assessed under high-resolution TEM (HRTEM), where lattice spacing measurements of $3.33 \AA$ and $2.25 \AA ̊$ were obtained, which are consistent with the $(012)^{26}$ and $(110)^{27}$ planes of elemental bismuth, respectively (Figure 3.1d). The crystallinity and phase were further confirmed with powder X- 
ray diffraction (PXRD), which showed a highly crystalline product that can be indexed to elemental bismuth (Figure 3.1e).
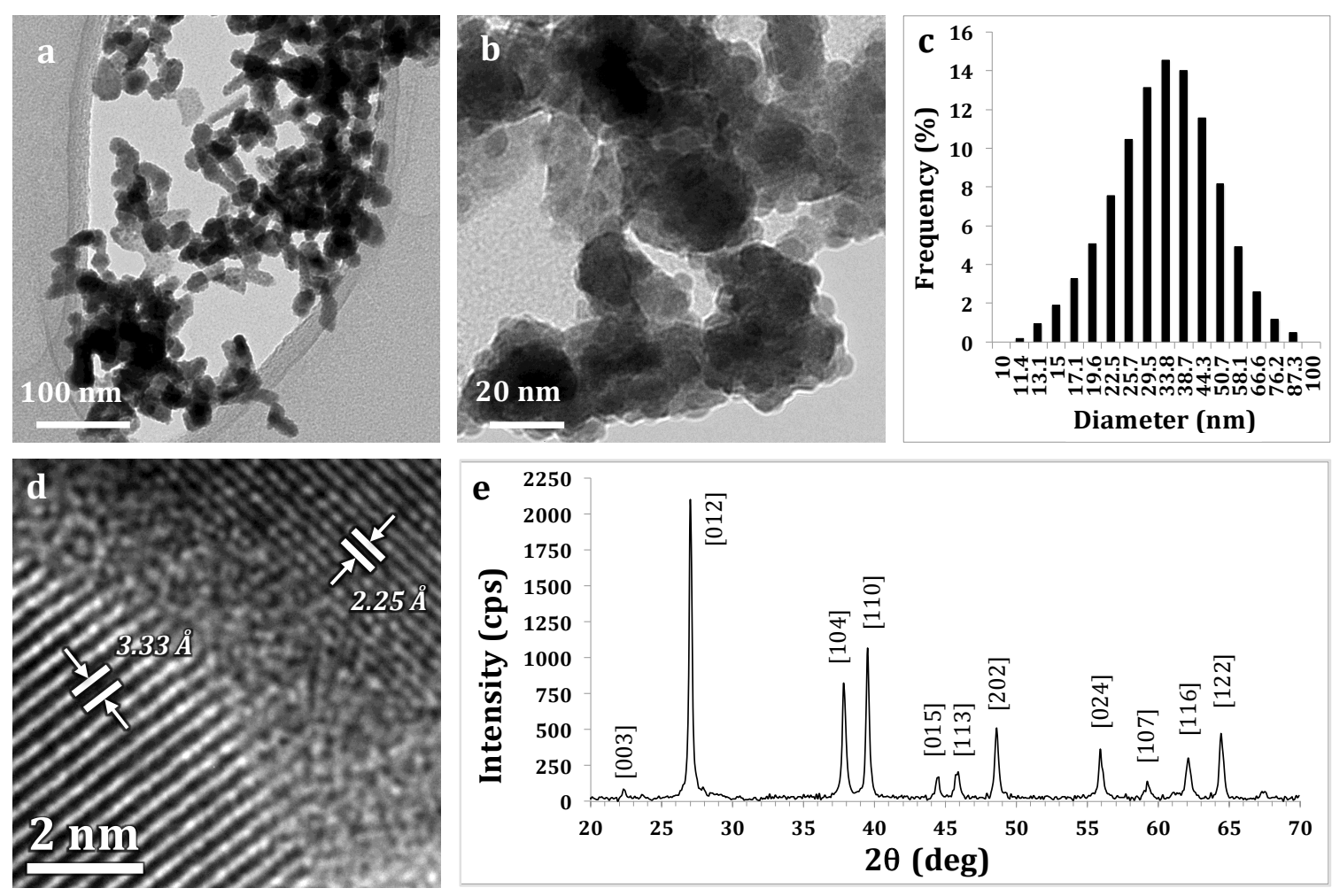

Figure 3.1: TEM images of Bi NPs synthesized in an aqueous solution of HMD (a \& b); c) DLS histogram of synthesized Bi NPs indicating an average hydrodynamic diameter of 33.4 $\mathrm{nm} \pm 12.5 \mathrm{~nm}$; d) HRTEM showing measured lattice spacings of $3.33 \AA$ and $2.25 \AA$, corresponding to the (012) and (110) planes of elemental bismuth, respectively; e) XRD pattern of a highly crystalline Bi NP powder.

In this synthesis, HMD was chosen as a surface-stabilizing agent for several reasons. First, it exhibits high water solubility due to the diamine functionality, which should ultimately result in a water-soluble product upon binding to the nanoparticle surfaces. Secondly, with a pKa of $\sim 11.2$, HMD would be tolerant to the elevated alkalinity of the synthetic environment. Lastly, between the terminal 
amines of HMD is a saturated hydrocarbon interior, which should act as an unreactive hydrophobic barrier between the nanoparticle surface and the highly oxidative, aqueous synthetic environment. A similar technique to mitigate surface oxidation was carried out in our lab with n-decane-terminated silicon nanoparticles (Si NPs), where the surfactant cetyltrimethylammonium bromide (CTAB), was used as a phase-transfer agent to make the hydrophobic Si NPs water-soluble. ${ }^{28}$ The hydrophobic tail of CTAB associated via van der Walls interactions with the decane on the NP surface, effectively generating a hydrophobic barrier, which protected the Si NPs from surface oxidation by limiting water access. We hypothesized that the aliphatic spacer between the terminal amines of HMD would act similarly to protect the Bi NP surfaces from oxidation, and subsequent dissolution, as was observed in our earlier synthetic work (see Chapter 2, Section 2.4).

Typically, post-synthetic workup to isolate Bi NPs from a reaction solution involves repeated centrifugation steps, which usually results in the loss of a large amount of product through decantation. Additionally, we've observed a greater extent of particle aggregation with prolonged centrifugation, which led to the necessity of finding an alternative method of particle isolation. With the HMD route, workup is made considerably easier. Bi NPs are isolated in a combination chemical/physical process, where particles are flocculated with the dropwise addition of a $0.1 \mathrm{M}$ solution of $\mathrm{HCl}$. The flocculate can then be easily isolated by brief centrifugation, and the particles can subsequently be re-suspended by the dropwise addition of $\mathrm{NaOH}$. 


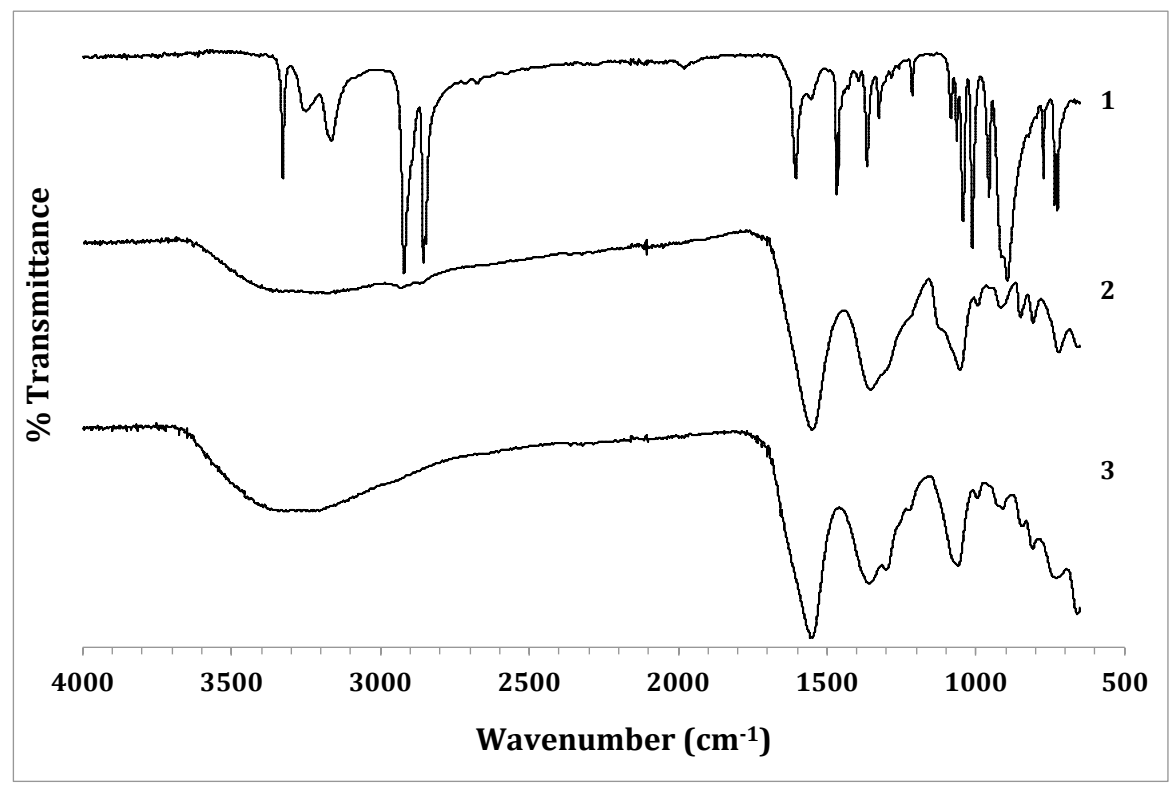

Figure 3.2: FTIR surface characterization of Bi NPs synthesized in the presence of HMD. Spectra are as follows: 1) hexamethylenediamine (HMD); 2) isolated and dried Bi NP flocculate; 3 ) dried and isolated insoluble $\mathrm{Bi}_{\mathrm{x}}(\mathrm{D}-\mathrm{TA})_{\mathrm{y}}$ precursor.

Isolating Bi NPs through the above method was thought to occur through the protonation of the terminal amines of HMD on the particle surface, forming an ammonium chloride salt. However, FTIR analysis was done to ascertain the chemical environment on the surface of the particles (Figure 3.2). The spectrum obtained for the flocculated particles (2) has many of the same features seen in the spectrum obtained for the $\mathrm{Bi}_{\mathrm{x}}(\mathrm{D}-\mathrm{TA})_{\mathrm{y}}$ precursor species (3) prior to addition of $\mathrm{NaOH}$, which suggests that D-TA is the dominant species on the NP surface. However, there also appear to be weak features of HMD (1) in the spectrum for the NP flocculate in the methylene $\left(-\mathrm{CH}_{2}\right)$ stretching region around $2800-2950 \mathrm{~cm}^{-1}$. These observations support the mechanism for particle isolation depicted in Scheme 3.1, where the terminal carboxylate groups of D-TA found on the particle surface are protonated 
through the addition of $\mathrm{HCl}$. Protonation of the carboxylate groups ultimately decreases the overall surface charge of particles, thereby diminishing their electrostatic stability and causing the particles to flocculate and fall out of solution. Additionally, this coincides with the acid/base chemistry of the $B_{x}(D-T A)_{y}$ precursor species, where under acidic conditions, the precursor is insoluble, but increasing the solution $\mathrm{pH}$ allows for complete precursor solubility. Further attempts to analyze the Bi NPs surface were carried out using proton nuclear magnetic resonance $\left({ }^{1} \mathrm{H}\right.$ NMR) spectroscopy, however, no information could be extracted from this analysis, as obtained spectra showed only signals for water and the deuterated solvent being used.

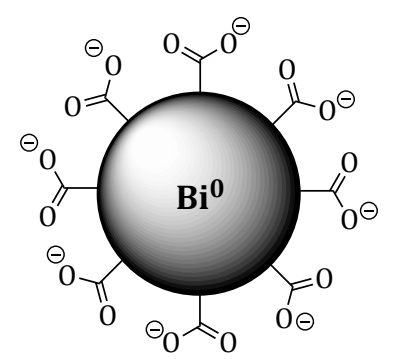

Colloidal Bi NPs

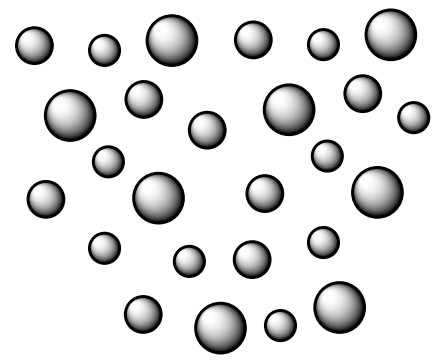

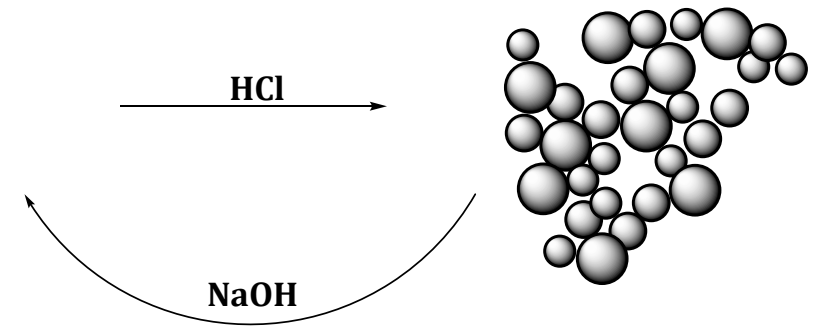

Scheme 3.1 Scheme depicting the method with which Bi NPs synthesized in the presence of HMD were isolated, using a combination chemical/physical approach.

Regardless of what species may be passivating the Bi NP surfaces, particles synthesized through this route were found to be highly stable to oxidative 
dissolution during and post synthesis. Bi NPs post-synthesis were found to stay colloidally stable in DI $\mathrm{H}_{2} \mathrm{O}$ following isolation for a period of several months. Eventually, the dark black colloids started to turn gray, likely due to surface oxidation, which resulted in particles aggregating and settling. However, this observed stability implies that these Bi NPs can be stored for prolonged periods of time and used for further applications and analysis.

\subsection{Conclusions}

To summarize, we report the synthesis of $33.4 \pm 12.5 \mathrm{~nm}$ Bi NPs through chemical reduction of a $\mathrm{Bi}_{\mathrm{x}}(\mathrm{D}-\mathrm{TA})_{\mathrm{y}}$ precursor with borane morpholine in the presence of HMD. HMD was initially thought to be the surface stabilizing species in the synthesis; however, it was observed by FTIR that the complexing agent, tartrate, was the predominant species on the particle surfaces. Although tartrate was not the intended surface species, its presence on the particle surfaces enables high watersolubility and the possibility for functionalization with other potential surface ligands. While the resulting Bi NPs may not show morphological uniformity, this synthesis does provide a low cost and sustainable approach toward aqueous, colloidally stable Bi NPs. 


\section{Terminal References}

\section{References from Chapter 1:}

[1] F. Dong, T. Xiong, Y. Sun, Z. Zhao, Y. Zhou, X. Feng, Z. Wu; Chem. Commun., 2014, $50,10386-10389$.

[2] K. Pothula, L. Tang, Z. Zha, Z. Wang; RSC Adv., 2015, 5, 83144-83148.

[3] F. Xia, X. Xu, X. Li, Lei Zhang, Li Zhang, H. Qiu, W. Wang, Y. Liu, J. Gao; Ind. Eng. Chem. Res., 2014, 53, 10576-10582.

[4] P. Gonzalez-Rodriguez, K. J. H. van den Nieuwenhuijzen, D. J. Schipper, J. E. ten Elshof; ACS Appl. Mater. Interfaces, 2016, 8, 7601-7606.

[5] J. Heremans, C. M. Thrush; Phys. Rev. B 1999, 59, 12579-12583.

[6] A. Boukai, K. Xu, J. R. Heath; Adv. Mater. 2006, 18, 864-869.

[7] X. Sun, Z. Zhang, M. S. Dresselhaus; Appl. Phys. Lett., 1999, 74, 4005-4007

[8] A. L. Brown, P. C. Naha, V. Benevides-Montes, H. I. Litt, A. M. Goforth, D. P. Cormode; Chem. Mater. 2014, 26, 2266-2274.

[9] Z. Li, J. Liu, Y. Hu, Z. Li, X. Fan, Y. Sun, F. Besenbacher, C. Chen, M. Yu; Biomaterials, 2017, 141, 284-295.

[10] B. Wei, X. Zhang, C. Zhang, T. Jiang, Y. Fu, C. Yu, S. Sun, X. Yan; Appl. Mater. Interfaces, 2016, 8, 12720-12726.

[11] P. Chhour, F. Cheheltani, P. C. Naha, H. I. Litt, V. A. Ferrari, D. P. Cormode; Design and Applications of Nanoparticles in Biomedical Imaging: Nanoparticles for Cardiovascular Imaging with CT; Springer, 2016, 357-384.

[12] S. D. Huang; Design and Applications of Nanoparticles in Biomedical Imaging: Bismuth-Based Nanoparticles for CT Imaging; Springer, 2016, 429-444. 
[13] O. Rabin, J. Manuel Perez, J. Grimm, G. Wojtkiewicz, R. Weissleder; Nat. Mater., 2006, 5, 118-122.

[14] D. Pan, E. Toessl, J. Schlomka, S. D. Caruthers, A. Senpan, M. J. Scott, J. S. Allen, H. Zhang, G. Hu, P. J. Gaffney, E. T. Choi, V. Rasche, S. A. Wickline, R. Proksa, G. M. Lanza; Angew. Chem., 2010, 122, 9829-9833.

[15] K. Ai, Y. Liu, J. Liu, Q. Yuan, Y. He, L. Lu; Adv. Mater. 2011, 23, 4886-4891.

[16] H. Lusic, M. W. Grinstaff; Chem. Rev., 2013, 113, 1641-1666.

[17] D. Kim, S. Park, J. H. Lee, Y. Y. Jeong, S. Jon; J. Am. Chem. Soc., 2007, 129, 76617665.

[18] Z. Liu, Z. Li, J. Liu, S. Gu, Q. Yuan, J. Ren, X. Qu; Biomaterials, 2012, 33, 67486757.

[19] A. S. Torres, P. J. Bonitatibus, R. E. Colborn, G. D. Goddard, P. F. FitzGerald, B. D. Lee, M. E. Marino; Investigative Radiology, 2012, 47, 578-587.

[20] P. J. Bonitatibus, A. S. Torres, B. Kandapallil, B. D. Lee, G. D. Goddard, R. E. Colborn, M. E. Marino; ACS Nano, 2012, 6, 6650-6658.

[21] J. F. Hainfeld, D. N. Slatkin, T. M. Focella, H. M. Smilowitz; The British Journal of Radiology, 2006, 79, 248-253.

[22] P. V. Dudin, P. R. Unwin, J. V. Macpherson; J. Phys. Chem. C 2010, 114, 1324113248.

[23] M. Behera, S. Ram; Appl. Nanosci. 2014, 4, 247-254.

[24] J. Polte, T. T. Ahner, F. Delissen, S. Sokolov, F. Emmerling, A. F. Thünemann, R. Kraehnert; J. Am. Chem. Soc. 2010, 132, 1296-1301.

[25] N. Zheng, J. Fan, G. D. Stucky; J. Am. Chem. Soc. 2006, 128, 6550-6551. 
[26] T. Sugimoto; Advances in Colloid and Interface Science, 1987, 28, 65-108.

[27] A. R. Tao, S. Habas, P. Yang; Small, 2008, 4, 310-325.

[28] R. J. Hunter; Zeta Potential in Colloid Science: Principles and Applications, $3^{\text {rd }}$ Ed.; Academic Press Inc., 1981.

[29] T. Cosgrove; Colloid Science: Principles, Methods and Applications, $2^{\text {nd }}$ Ed.;

Wiley, 2010.

[30] W. J. I. DeBenedetti, S. -K. Chiu, C. M. Radlinger, R. J. Ellison, B. A. Manhat, J. Z.

Zhang, J. Shi, A. M. Goforth; J. Phys. Chem. C; 2015, 119, 9595-9608.

[31] X. Peng, L. Manna, W. Yang, J. Wickham, E. Scher, A. Kadavanich, A. P. Alivisatos; Nature, 2000, 404, 59-61.

[32] L. Manna, E. C. Scher, A. P. Alivisatos; J. Am. Chem. Soc., 2000, 122, 1270012706.

[33] Y. Jun, M. F. Casula, J. Sim, S. Y. Kim, J. Cheon, A. P. Alivisatos; J. Am. Chem. Soc. 2003, 125, 15981-15985.

[34] F. E. Kruis, H. Fissan, A. Peled; J. Aerosol Sci., 1998, 29, 511-535.

[35] J. Singh, D. E. Wolfe; J. Materials Sci., 2005, 40, 1-26.

[36] M. T. Swihart; Colloid and Interface Sci., 2003, 8, 127-133.

[37] D. B. Goia, E. Matijević; New J. Chem. 1998, 22, 1203-1215.

[38] S. G. Bratsch; J. Phys. Chem. Ref. Data, 1989, 18, 1-21.

[39] J. S. Son, K. Park, M. Han, C. Kang, S. Park, J. Kim, W. Kim, S. Kim, T. Hyeon; Angew. Chem. 2011, 123, 1399-1402.

[40] G. Carotenuto, C. L. Hison, F. Capezzuto, M. Palomba, P. Perlo, P. Conte; J. Nanopart. Res. 2009, 11, 1729-1738. 
[41] L. Balan, R. Schneider, D. Billaud, Y. Fort, J. Ghanbaja; Nanotechnology 2004, 15, 940-944.

[42] J. Wang, X. Wang, Q. Peng, Y. Li; Inorg. Chem. 2004, 43, 7552-7556.

[43] F. Wang, R. Tang, H. Yu, P. C. Gibbons, W. E. Buhro; Chem. Mater. 2008, 20, 3656-3662.

[44] J. S. Son, K. Park, M. Han, C. Kang, S. Park, J. Kim, W. Kim, S. Kim, T. Hyeon; Angew. Chem. 2011, 123, 1399-1402.

[45] J. Wang, X. Wang, Q. Peng, Y. Li; Inorg. Chem. 2004, 43, 7552-7556.

[46] S. C. Warren, A. C. Jackson, Z. D. Cater-Cyker, F. J. DiSalvo, U. Wiesner; J. Am. Chem. Soc. 2007, 129, 10072-10073.

[47] R. N. Grass, W. J. Stark; J. Nano. Res. 2006, 8, 729-736.

[48] Y. Wang, Y. Xia; Nano Lett. 2004, 4, 2047-2050.

[49] K. Wegner, B. Walker, S. Tsantilis, S. E. Pratsinis; Chem. Eng. Sci. 2002, 57, 17531762.

[50] B. Gersten; Chemfiles Volume 5 Article 13.

[51] Y. Wang, J. Zhao, X. Zhao, L. Tang, Y. Li, Z. Wang; Mat. Res. Bulletin 2009, 44, 220-223.

[52] D. Ma, J. Zhao, Y. Zhao, X. Hao, L. Li, L. Zhang, Y. Lu, C. Yu; Colloids and Surfaces A: Physiochem. Eng. Aspects 2012, 395, 276-283.

[53] A. L. Brown, A. M. Goforth; Chem. Mater. 2012, 24, 1599-1605.

\section{References from Chapter 2:}

[1] M. A. El-Sayed, Acc. Chem. Res. 2001, 34, 257-264. 
[2] M. Valden, X. Lai, D. W. Goodman, Science 1998, 281, 1647-1650.

[3] C. J. Murphy, Science 2002, 298, 2139-2141.

[4] H. S. Choi, W. Liu, P. Misra, E. Tanaka, J. P. Zimmer, B. I. Ipe, M. G. Bawendi, J. V. Frangioni; Nat. Biotechnol. 2007, 25, 1165-1170.

[5] A. Jakhmola, N. Anton, T. F. Vandamme; Adv. Healthcare Mater. 2012, 1, 413-431.

[6] D. P. Cormode, P. Naha, Z. A. Fayad; Contrast Media Mol. Imaging 2014, 9, 37-52.

[7] H. Lusic, M. W. Grinstaff; Chem. Rev. 2013, 113, 1641-1666.

[8] 0. Rabin, J. M. Perez, J. Grimm, G. Wojtkiewicz, R. Weissleder; Nat. Mater. 2006, 5, 118-122.

[9] J. M. Kinsella, R. E. Jimenez, P. P. Karmali, A. M. Rush, V. R. Kotamraju, N. C. Gianneschi, E. Ruoslahti, D. Stupack, M. J. Sailor; Angew. Chem., Int. Ed. 2011, 50, 12308-12311.

[10] I. E. Allijn, W. Leong, J. Tang, A. Gianella, A. J. Mieszawska, F. Fay, G. Ma, S. Russell, C. B. Callo, R. E. Gordon, E. Korkmaz, J. A. Post, Y. Zhao, H. C. Gerritsen, G. Storm, A. Thran, R. Proksa, H. Daerr, V. Fuster, E. A. Fisher, Z. A. Fayad, W. J. Mulder, D. P. Cormode; ACS Nano 2013, 7, 9761-9770.

[11] P. J. Bonitatibus, A. S. Torres, B. Kandapallil, B. D. Lee, G. D. Goddard, R. E. Colborn, M. E. Marino; ACS Nano 2012, 6, 6650-6658. [12] A. S. Torres, P. J. Bonitatibus, R. E. Colborn, G. D. Goddard, P. F. FitzGerald, B. D. Lee, M. E. Marino; Invest. Radiol. 2012, 47, 578-587. [13] C. Alric, J. Taleb, G. Le Duc, C. Mandon, C. Billotey, A. LeMeur-Herland, T. Brochard, F. Vocanson, M. Janier, P. Perriat, S. Roux, O. Tillement; J. Am. Chem. Soc. 2008, 130, 5908-5915. 
[14] S. -W. Chou, Y. -H. Shau, P. -C. Wu, Y. -S. Yang, D. -B. Shieh, C. -C. Chen; J. Am. Chem. Soc. 2010, 132, 13270-13278.

[15] M. H. Oh, N. Lee, H. Kim, S. P. Park, Y. Piao, J. Lee, S. W. Jun, W. K. Moon, S. H. Choi, T. Hyeon; J. Am. Chem. Soc. 2011, 133, 5508-5515.

[16] N. Lee, H. R. Cho, M. H. Oh, S. H. Lee, K. Kim, B. H. Kim, K. Shin, T. Y. Ahn, J. W. Choi, Y. W. Kim, S. H. Choi, T. Hyeon; J. Am. Chem. Soc. 2012, 134, 10309-10312. [17] F. Dong, T. Xiong, Y. Sun, Z. Zhao, Y. Zhou, X. Feng, Z. Wu; Chem. Commun. 2014, 50, 10386-10389.

[18] K. Pothula, L. Tang, Z. Zha, Z. Wang; RSC Adv. 2015, 5, 83144-83148.

[19] F. Xia, X. Xu, X. Li, Lei Zhang, Li Zhang, H. Qiu, W. Wang, Y. Liu, J. Gao; Ind. Eng. Chem. Res. 2014, 53, 10576-10582.

[20] J. Heremans, C. M. Thrush; Phys. Rev. B 1999, 59, 12579-12583.

[21] A. Boukai, K. Xu, J. R. Heath; Adv. Mater. 2006, 18, 864-869.

[22] D. D. Fanfair, B. A. Korgel; Cryst. Growth Des. 2005, 5, 1971-1976.

[23] A. L. Brown, P. C. Naha, V. Benevides-Montes, H. I. Litt, A. M. Goforth, D. P. Cormode; Chem. Mater. 2014, 26, 2266-2274

[24] Z. Li, J. Liu, Y. Hu, Z. Li, X. Fan, Y. Sun, F. Besenbacher, C. Chen, M. Yu; Biomaterials 2017, 141, 284-295.

[25] B. Wei, X. Zhang, C. Zhang, T. Jiang, Y. Fu, C. Yu, S. Sun, X. Yan; Appl. Mater. Interfaces 2016, 8, 12720-12726.

[26] P. Chhour, F. Cheheltani, P. C. Naha, H. I. Litt, V. A. Ferrari, D. P. Cormode; Design and Applications of Nanoparticles in Biomedical Imaging: Nanoparticles for Cardiovascular Imaging with CT; Springer 2016, 357-384. 
[27] S. D. Huang; Design and Applications of Nanoparticles in Biomedical Imaging: Bismuth-Based Nanoparticles for CT Imaging; Springer 2016, 429-444.

[28] O. Rabin, J. Manuel Perez, J. Grimm, G. Wojtkiewicz, R. Weissleder; Nat. Mater. $2006,5,118-122$.

[29] D. Pan, E. Toessl, J. Schlomka, S. D. Caruthers, A. Senpan, M. J. Scott, J. S. Allen, H. Zhang, G. Hu, P. J. Gaffney, E. T. Choi, V. Rasche, S. A. Wickline, R. Proksa, G. M. Lanza; Angew. Chem. 2010, 122, 9829-9833.

[30] K. Ai, Y. Liu, J. Liu, Q. Yuan, Y. He, L. Lu; Adv. Mater. 2011, 23, 4886-4891.

[31] Bismuth salts (https://www.sigmaaldrich.com/materials-science/materialscience-products.html?TablePage=19295358)

[32] N. N. Greenwood, A. Earnshaw; Chemistry of the Elements, 2nd Ed.;

Butterworth-Heinemann: Oxford, U.K., 1998, 547-600.

[33] J. Li, F. Jiang, B. Yang, X.-R. Song, Y. Liu, H.-H. Yang, D.-R. Cao, W.-R. Shi, G.-N. Chen; Sci. Rep. 2013, 3, 1-7.

[34] G. Glen, N. Burford; Chem. Rev. 1999, 99, 2601-2657.

[35] P. J. Sadler, H. Li, H. Sun; Coord. Chem. Rev. 1999, 185-186, 689-709.

[36] C. Lopez-Chaves, J. Soto-Alvaredo, M. Montes-Bayon, J. Bettmer, J. Llopis, C. Sanchez-Gonzalez; Nanomedicine 2017, 17, 30158-30162.

[37] C. Rambanapasi, J. R. Zeevaart, H. Buntting, C. Bester, D. Kotze, R. Hayeshi, A. Grobler; Molecules 2016, 21, 763-775.

[38] J. S. Son, K. Park, M. Han, C. Kang, S. Park, J. Kim, W. Kim, S. Kim, T. Hyeon; Angew. Chem. 2011, 123, 1399-1402. 
[39] G. Carotenuto, C. L. Hison, F. Capezzuto, M. Palomba, P. Perlo, P. Conte; J. Nanopart. Res. 2009, 11, 1729-1738.

[40] L. Balan, R. Schneider, D. Billaud, Y. Fort, J. Ghanbaja; Nanotechnology 2004, 15, 940-944.

[41] J. Wang, X. Wang, Q. Peng, Y. Li; Inorg. Chem. 2004, 43, 7552-7556.

[42] F. Wang, R. Tang, H. Yu, P. C. Gibbons, W. E. Buhro; Chem. Mater. 2008, 20, 3656-3662.

[43] J. S. Son, K. Park, M. Han, C. Kang, S. Park, J. Kim, W. Kim, S. Kim, T. Hyeon; Angew. Chem. 2011, 123, 1399-1402.

[44] J. Wang, X. Wang, Q. Peng, Y. Li; Inorg. Chem. 2004, 43, 7552-7556.

[45] S. C. Warren, A. C. Jackson, Z. D. Cater-Cyker, F. J. DiSalvo, U. Wiesner; J. Am. Chem. Soc. 2007, 129, 10072-10073.

[46] Y. Wang, J. Zhao, X. Zhao, L. Tang, Y. Li, Z. Wang; Mat. Res. Bulletin 2009, 44, 220-223.

[47] D. Ma, J. Zhao, Y. Zhao, X. Hao, L. Li, L. Zhang, Y. Lu, C. Yu; Colloids and Surfaces A: Physiochem. Eng. Aspects 2012, 395, 276-283.

[48] A. L. Brown, A. M. Goforth; Chem. Mater. 2012, 24, 1599-1605.

[49] S. B. Kalidindi, U. Sanyal, B. R. Jagirdar; ChemSusChem 2011, 4, 317-324.

[50] C. J. Hiatt; "Elemental Bismuth Nanoparticles : Mechanistic Studies Concerning Reduction of a Bi(III) Precursor Leading to Nanoparticle Formation in a Bottom-Up, High Payload Synthetic Approach" 2014, University Honors Theses. Paper 107. http://pdxscholar.library.pdx.edu/honorstheses/107. [51] Fehling's test (http://www.chem.ucla.edu/ harding/IGOC/F/fehlings_test.ht 
$\mathrm{ml)}$ for aldehydes and Tollens' test (https://eic.rsc.org/exhibition-chemistry/thesilver-mirror-test/2020077.article) for aldehydes, which are similar to the $\alpha$-Dglucose reduction of $\mathrm{Bi}$ (III) (to form elemental $\mathrm{Bi}$ ) in the presence of $\mathrm{OH}^{-}$, used extensively in carbohydrate chemistry.

[52] F. Franks, P. J. Lillford, G. Robinson; J. Chem. Soc., Faraday Trans. 1 1989, 85(8), $2417-2426$.

[53] S. Panigrahi, S. Kundu, S. K. Ghosh, S. Nath, T. Pal; J. Nanoparticle Research $2004,6,411-414$.

[54] J. Liu, G. Qin, P. Raveendran, Y. Ikkushima; Chem. Eur. J. 2006, 12, 2131-2138.

[55] M. Jin, G. He, H. Zhang, J. Zeng, Z. Xie, Y. Xia; Angew. Chem. Int. Ed. 2011, 50, 10560-10564.

[56] J. In, I. Yoon, K. Seo, J. Park, J. Choo, Y. Lee, B. Kim; Chem. Eur. J. 2011, 17, 13041309.

[57] J. V. Jokerst, T. Lobovkina, R. N. Zare, S. S. Gambhir; Nanomedicine (Lond). 2011, 6, 715-738.

\section{References from Chapter 3:}

[1] F. Dong, T. Xiong, Y. Sun, Z. Zhao, Y. Zhou, X. Feng, Z. Wu; Chem. Commun. 2014, 50, 10386-10389.

[2] K. Pothula, L. Tang, Z. Zha, Z. Wang; RSC Adv. 2015, 5, 83144-83148.

[3] F. Xia, X. Xu, X. Li, Lei Zhang, Li Zhang, H. Qiu, W. Wang, Y. Liu, J. Gao; Ind. Eng. Chem. Res. 2014, 53, 10576-10582. 
[4] J. Heremans, C. M. Thrush; Phys. Rev. B 1999, 59, 12579-12583.

[5] A. Boukai, K. Xu, J. R. Heath; Adv. Mater. 2006, 18, 864-869.

[6] D. D. Fanfair, B. A. Korgel; Cryst. Growth Des. 2005, 5, 1971-1976.

[7] A. L. Brown, P. C. Naha, V. Benevides-Montes, H. I. Litt, A. M. Goforth, D. P. Cormode; Chem. Mater. 2014, 26, 2266-2274

[8] Z. Li, J. Liu, Y. Hu, Z. Li, X. Fan, Y. Sun, F. Besenbacher, C. Chen, M. Yu; Biomaterials $2017,141,284-295$.

[9] B. Wei, X. Zhang, C. Zhang, T. Jiang, Y. Fu, C. Yu, S. Sun, X. Yan; Appl. Mater. Interfaces 2016, 8, 12720-12726.

[10] P. Chhour, F. Cheheltani, P. C. Naha, H. I. Litt, V. A. Ferrari, D. P. Cormode; Design and Applications of Nanoparticles in Biomedical Imaging: Nanoparticles for Cardiovascular Imaging with CT; Springer 2016, 357-384.

[11] S. D. Huang; Design and Applications of Nanoparticles in Biomedical Imaging: Bismuth-Based Nanoparticles for CT Imaging; Springer 2016, 429-444.

[12] O. Rabin, J. Manuel Perez, J. Grimm, G. Wojtkiewicz, R. Weissleder; Nat. Mater. $2006,5,118-122$.

[13] D. Pan, E. Toessl, J. Schlomka, S. D. Caruthers, A. Senpan, M. J. Scott, J. S. Allen, H. Zhang, G. Hu, P. J. Gaffney, E. T. Choi, V. Rasche, S. A. Wickline, R. Proksa, G. M. Lanza; Angew. Chem. 2010, 122, 9829-9833.

[14] K. Ai, Y. Liu, J. Liu, Q. Yuan, Y. He, L. Lu; Adv. Mater. 2011, 23, 4886-4891.

[15] H. Lusic, M. W. Grinstaff; Chem. Rev. 2013, 113, 1641-1666.

[16] P. J. Sadler, H. Li, H. Sun; Coordination Chemistry Reviews 1999, 185-186, 689709. 
[17] G. G. Briand, N. Burford; Chem. Rev. 1999, 2601-2657.

[18] G. G. Briand, N. Burford; Coordination complexes of bismuth (III) involving organic ligands with pnictogen or chalcogen donors; Advances in Inorganic Chemistry; Academic Press, 2000, Vol. 50, 285-357. [19] F. Xia, X. Xu, X. Li, Lei Zhang, Li Zhang, H. Qiu, W. Wang, Y. Liu, J. Gao; Ind. Eng. Chem. Res. 2014, 53, 10576-10582.

[20] M. Yarema, M. V. Kovalenko, G. Hesser, D. V. Talapin, W. Heiss; J. Am. Chem. Soc. 2010, 132, 15158-15159.

[21] F. Wang, R. Tang, H. Yu, P. C. Gibbons, W. E. Buhro; Chem. Mater. 2008, 20, 3656-3662.

[22] H. Yu, P. C. Gibbons, K. F. Kelton, W. E. Buhro; J. Am. Chem. Soc. 2001, 123, 91989199.

[23] M. He, L. Protesescu, R. Caputo, F. Krumeich, M. V. Kovalenko; Chem. Mater. $2015,27,635-647$.

[24] H. Liu, S. Zhang, P. Zhang, Y. Zhang; Proceedings of the $14^{\text {th }}$ IFToMM World Congress 2015, 14, 547-555.

[25] J. Chen, L. -M. Wu, L. Chen; Inorg. Chem. 2007, 46, 586-591.

[26] S. -K. Chiu, B. A. Manhat, W. J. I. DeBenedetti, A. L. Brown, K. Fichter, T. Vu, M. Eastman, J. Jiao, A. M. Goforth; J. Mater. Res. 2013, 28, 216-230.

[27] A. L. Brown, A. M. Goforth; Chem. Mater. 2012, 24, 1599-1605.

[28] Z. Li, J. Liu, Y. Hu, Z. Li, X. Fan, Y. Sun, F. Besenbacher, C. Chen, M. Yu; Biomaterials 2017, 141, 284-295. 


\section{Appendix}

\section{Supporting Information}

Chapter 2 - Additional FTIR Spectra

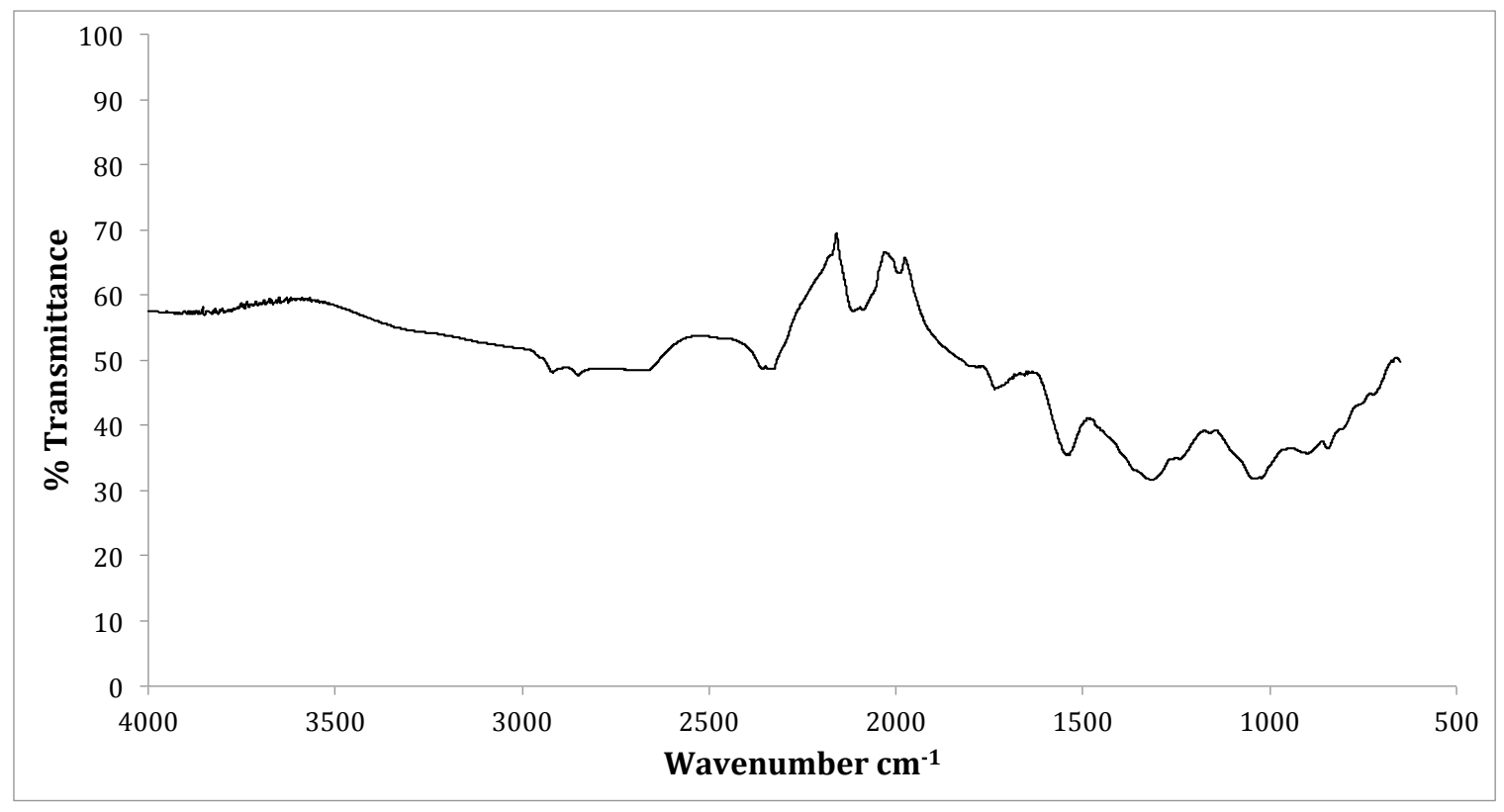

Figure S1: Representative FTIR spectrum of Bi NPs synthesized using glucose as the sole reducing agent, presenting broad absorption features over two different regions, ranging from $4000-2150 \mathrm{~cm}^{-1}$ and $2000-650 \mathrm{~cm}^{-1}$ (Chapter 2 Section 2.4.1). Clear absorption bands can be seen at $1720 \mathrm{~cm}^{-1}, 1535 \mathrm{~cm}^{-1}, 1308 \mathrm{~cm}^{-1}$, and $1035 \mathrm{~cm}^{-1}$ within the broadband absorbance in the finger print region, perhaps indicating the presence of a carboxylate group. 


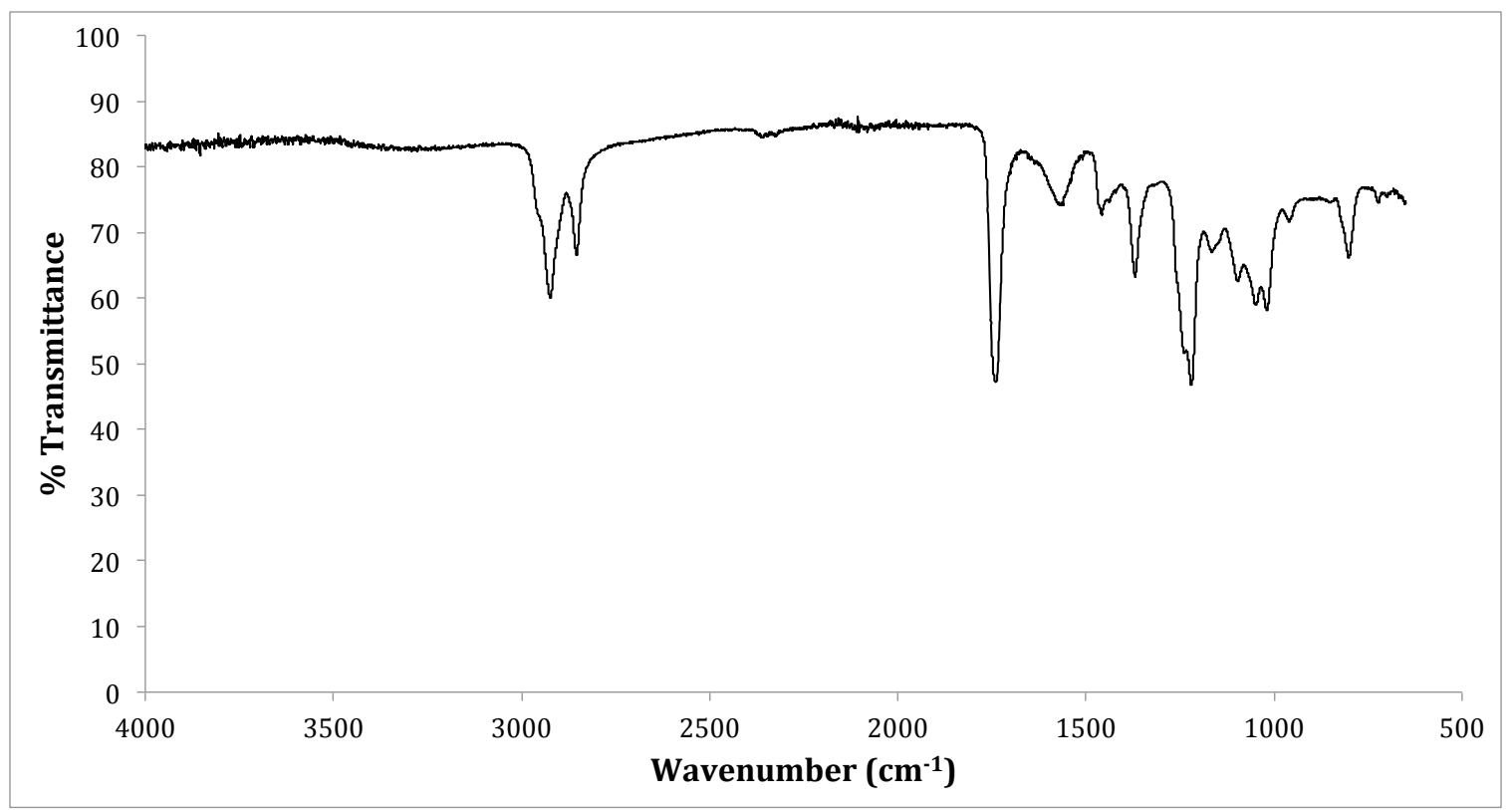

Figure S2: FTIR spectrum of Bi NPs synthesized in the presence of PEG 300 using borane morpholine as the reducing agent (Chapter 2 Section 2.4.3). A slight absorption band from $3500-3050 \mathrm{~cm}^{-1}$ in correlation with the strong absorption bands at $2922 \mathrm{~cm}^{-1}$ and $2852 \mathrm{~cm}^{-1}$ imply the presence of PEG 300 on the surface of the crystalline aggregate nanoclusters. Absorption bands at $1446 \mathrm{~cm}^{-1}$ and $1367 \mathrm{~cm}^{-}$ ${ }^{1}$ are also observed, which are consistent with the reference spectrum for PEG 300. However, additional bands are observed at $1734 \mathrm{~cm}^{-1}$ and $1558 \mathrm{~cm}^{-1}$, which might indicate the presence of a carboxylic acid/carboxylate group. 


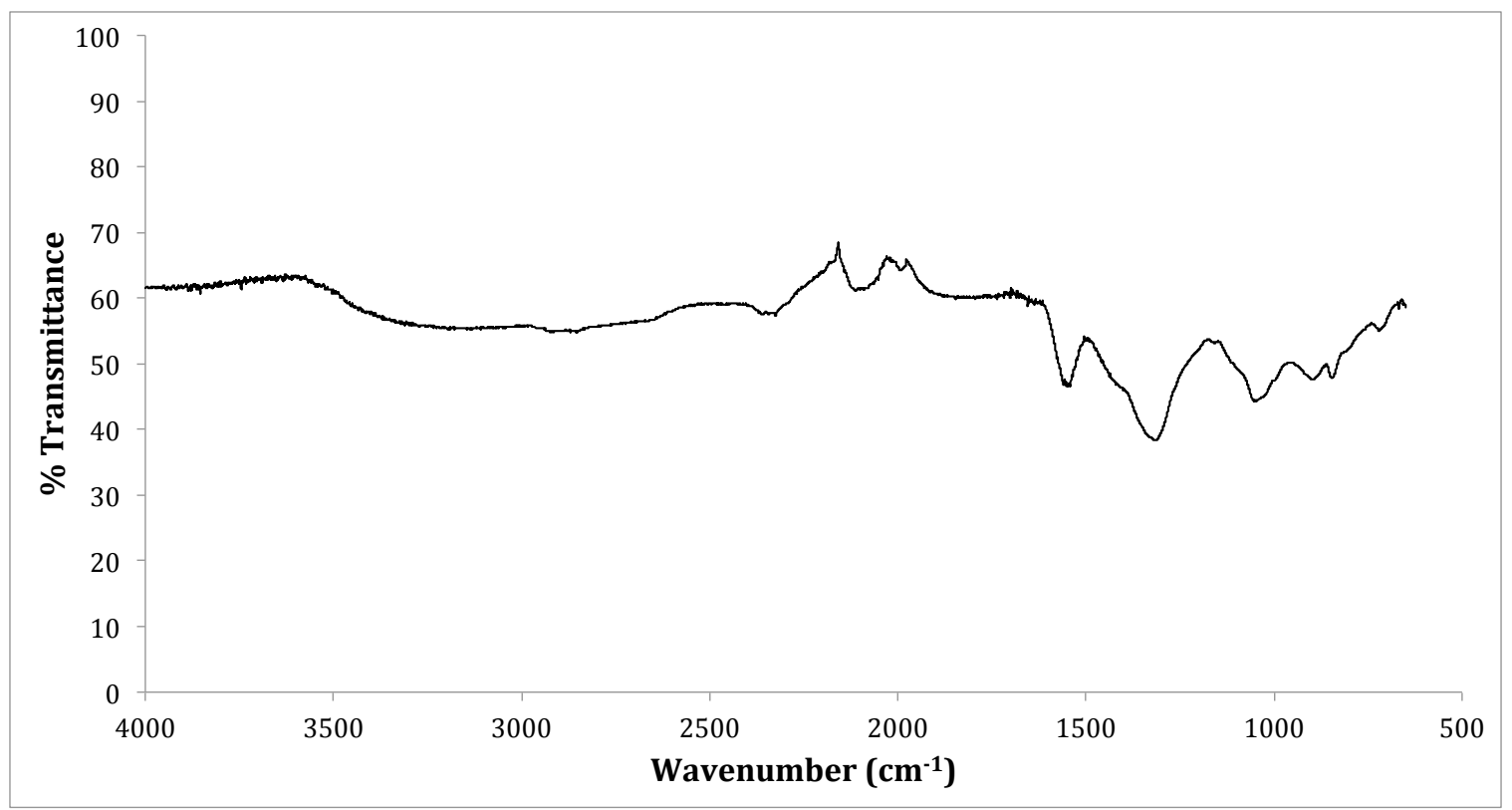

Figure S3: FTIR spectrum of particle colloid that underwent oxidative dissolution and regrew through incubation of the dissolved colloid (Chapter 2 Section 2.4.3). The entire spectrum appears to show broadband absorbance with several distinct absorption bands at $1539 \mathrm{~cm}^{-1}, 1302 \mathrm{~cm}^{-1}$, and $1027 \mathrm{~cm}^{-1}$, similar to the spectrum for particles obtained through the route using glucose as the sole reducing agent. 